\title{
ON THE CONTROL OF THE MOTION OF A BOAT
}

\author{
OLIVIER GLASS AND LIONEL ROSIER
}

\begin{abstract}
In this paper we study the control of the motion of a boat, viewed as a rigid body $S$ with one axis of symmetry, which is surrounded by an inviscid incompressible fluid filling $\mathbb{R}^{2} \backslash S$. We take as control input the flow of the fluid through a part of the boundary of the boat. We prove that the position, orientation, and velocity of the boat are locally controllable with a bidimensional control input, even if the flow displays some vorticity.
\end{abstract}

\section{INTRODUCTION}

The control of boats or submarines has attracted the attention of the mathematical community from a long time (see e.g. $[14,15,16,2]$.) In most of the papers devoted to that issue, the fluid is assumed to be inviscid, incompressible and irrotational, and the rigid body is supposed to have an elliptic shape. On the other hand, to simplify the model, the control is often assumed to appear in a linear way in a finite-dimensional system describing the dynamics of the rigid body, the so-called Kirchhoff laws.

A large vessel (e.g. a freighter) presents often one tunnel thruster built into the bow to make docking easier. The aim of this paper is to provide some accurate model of a boat controlled by two propellers, the one displayed in a transversal bowthruster at the bow of the ship, the other one placed at the stern of the boat (see Figure 1), and to give a rigorous analysis of the control properties of such a system.

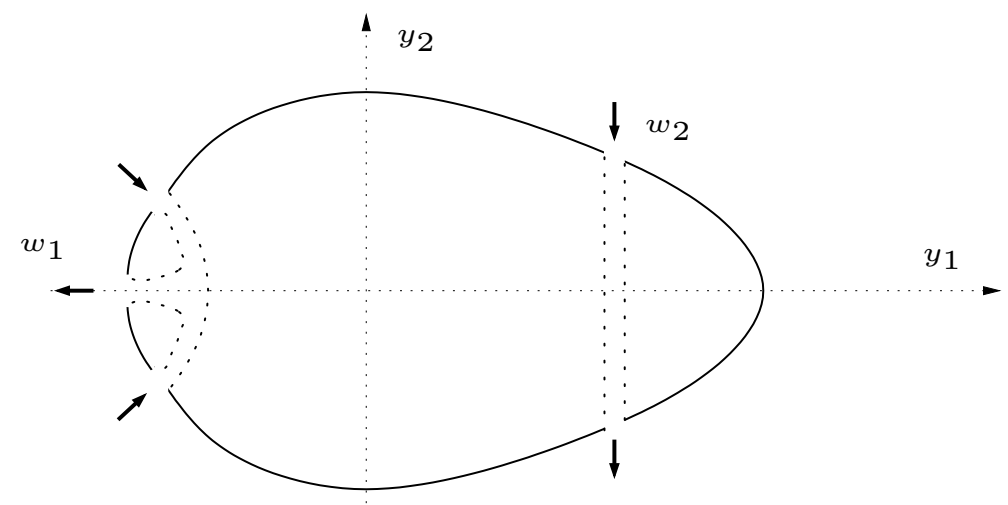

FiguRE 1. Forward propulsion by a propeller

1991 Mathematics Subject Classification. 35Q35, 76B03, 76B99.

Key words and phrases. Euler equations, fluid-rigid body interaction, exterior domain, classical solutions. 
The fluid, still inviscid and incompressible, will no longer be assumed to be irrotational (i.e., the vorticity may not vanish everywhere), and the only geometric assumption for the shape of the boat will be the existence of one axial symmetry.

To be more precise, we consider a boat, represented by a rigid body with one axis of symmetry occupying a bounded, simply connected open set $S(t) \subset \mathbb{R}^{2}$ of class $C^{\infty}$ and which is surrounded by a homogeneous incompressible perfect fluid. We denote by $\Omega(t)=\mathbb{R}^{2} \backslash \overline{S(t)}$ the domain occupied by the fluid, and write merely $S=S(0)$ and $\Omega=\Omega(0)$ for the domains occupied respectively by the rigid body and the fluid at $t=0$. The equations for the dynamics of the system fluid + rigid body read then

$$
\begin{array}{cc}
\frac{\partial u}{\partial t}+(u \cdot \nabla) u+\nabla p=0, & t \in[0, T], x \in \Omega(t), \\
\operatorname{div} u=0, & t \in[0, T], x \in \Omega(t), \\
u \cdot n=\left(h^{\prime}+r(x-h)^{\perp}\right) \cdot n+w(t, x), & t \in[0, T], x \in \partial \Omega(t), \\
\lim _{|x| \rightarrow \infty} u(t, x)=0, & \\
m h^{\prime \prime}=\int_{\partial \Omega(t)} p n d \sigma, & t \in[0, T], \\
J r^{\prime}=\int_{\partial \Omega(t)}(x-h)^{\perp} \cdot p n d \sigma, & t \in[0, T], \\
\theta^{\prime}=r & t \in[0, T], \\
(h(0), \theta(0))=\left(h_{0}, \theta_{0}\right) \in \mathbb{R}^{2} \times \mathbb{R},\left(h^{\prime}(0), r(0)\right)=\left(h_{1}, r_{0}\right) \in \mathbb{R}^{2} \times \mathbb{R} .
\end{array}
$$

In the above equations, $u$ (resp. $p$ ) is the velocity field (resp. the pressure) of the fluid, $h$ denotes the position of the center of mass of the solid, $\theta$ is the angle between some axis linked to the rigid body and a given fixed axis, and $r$ denotes the angular velocity. The positive constants $m$ and $J$, which denote respectively the mass and the moment of inertia of the rigid body, are defined as

$$
m=\int_{S} \rho(x) d x, \quad J=\int_{S} \rho(x)|x|^{2} d x,
$$

where $\rho(\cdot)$ denotes the density of the rigid body. The vector $n$ is the outward unit vector to $\partial \Omega(t)$, so that $\tau=-n^{\perp}=\left(n_{2},-n_{1}\right)$ is a unit tangent vector to $\partial \Omega(t)$. Finally, the term $w(t, x)$, which stands for the flow through the boundary of the rigid body, is taken as control input. Its support will be strictly included in $\partial \Omega(t)$, and actually only a finite dimensional control input will be considered here (see below (1.13) for the precise form of the control term $w(t, x))$.

When no control is applied (i.e. $w(t, x)=0$ ), then the existence and uniqueness of strong solutions to (1.1)-(1.9) was obtained in [18] for a ball, and in [19] for a rigid body $S$ of arbitrary form. The result in [18] was extended to any dimension in [20] (in that paper, the issue of the persistence of regularity is also studied). We also refer to [10] for the situation when $\Omega(t)=\Omega_{0} \backslash \overline{S(t)}$, with $\Omega_{0}$ a bounded open set in $\mathbb{R}^{3}$, and for the issue of the analyticity in time. The detection of the rigid body $S(t)$ from partial measurements of the fluid velocity has been tackled in [5] when $\Omega(t)=\Omega_{0} \backslash \overline{S(t)}\left(\Omega_{0} \subset \mathbb{R}^{2}\right.$ still being a bounded cavity) and in [4] when $\Omega(t)=\mathbb{R}^{2} \backslash \overline{S(t)}$.

Here, we are interested in the control properties of (1.1)-(1.9). The controllability of Euler equations has been established in 2D (resp. in 3D) in [6] (resp. in [9]) Note, however, that there is no hope here to control both the fluid and the rigid body motion. Indeed, 
$\Omega(t)$ is an exterior domain, and the vorticity is transported by the flow with a finite speed propagation, so that it is not affected (at any given time) far from the boat. Therefore, we will deal with the control of the motion of the rigid body only. As the state of the rigid body is described by a vector in $\mathbb{R}^{6}$, namely $\left(h, \theta, h^{\prime}, r\right)$, it is natural to consider a finite-dimensional control input.

Note also that since the fluid is flowing through a part of the boundary of the rigid body, one more boundary condition is needed to ensure the uniqueness of the solution of (1.1)-(1.9) (see [11], [12]). In dimension two, one can impose the value of the vorticity $\omega(t, x):=\operatorname{curl} v(t, x)$ on the inflow section of $\partial \Omega(t)$; that is, one can set

$$
\omega(t, x)=\omega_{0}(t, x) \quad \text { for } w(t, x)<0
$$

where $\omega_{0}(t, x)$ is a given function.

In order to write the equations of the fluid in a fixed domain, we perform a change of coordinates. We set

$$
\theta(t)=\theta_{0}+\int_{0}^{t} r(s) d s, \quad Q(\theta)=\left(\begin{array}{cc}
\cos \theta & -\sin \theta \\
\sin \theta & \cos \theta
\end{array}\right)
$$

and

$$
x=Q(\theta(t)) y+h(t),
$$

where $x$ (resp. $y$ ) represents the vector of coordinates of a point in a fixed frame (respectively in a frame linked to the rigid body). Note that, at any given time $t, y$ ranges over the fixed domain $\Omega$ when $x$ ranges over $\Omega(t)$. Next, we introduce the functions

$$
\left\{\begin{aligned}
v(t, y) & :=Q(\theta(t))^{*} u(t, Q(\theta(t)) y+h(t)), \\
q(t, y) & :=p(t, Q(\theta(t)) y+h(t)) \\
l(t) & :=Q(\theta(t))^{*} \dot{h}(t)
\end{aligned}\right.
$$

where ${ }^{*}=d / d t$ and ${ }^{*}$ means transpose. Finally, we assume that the control takes the form

$$
w(t, x)=w(t, Q(\theta(t)) y+h(t))=\sum_{1 \leqslant j \leqslant m} w_{j}(t) \chi_{j}(y),
$$

where $m \in \mathbb{N}^{*}$ stands for the number of independent inputs, and $w_{j}(t) \in \mathbb{R}$ is the control input associated with the function $\chi_{j} \in C^{\infty}(\partial \Omega)$. Often, the functions $\chi_{j}$ have disjoint supports, i.e.

$$
\chi_{j}(y) \chi_{k}(y)=0 \quad \forall y \in \partial \Omega, \forall j \neq k,
$$

but we shall not make this hypothesis thereafter. To ensure the conservation of the mass of the fluid, we impose the relation

$$
\int_{\partial \Omega} \chi_{j}(y) d \sigma=0 \quad \text { for } 1 \leqslant j \leqslant m .
$$

Finally, we assume that the solid is symmetric with respect to the $y_{1}$-axis (see Figure 2), i.e.

$$
\left(y_{1}, y_{2}\right) \in S \Rightarrow\left(y_{1},-y_{2}\right) \in S,
$$

and that the functions $\chi_{j}$ fulfill the following symmetry properties

$$
\begin{aligned}
\chi_{1}\left(y_{1},-y_{2}\right)=\chi_{1}\left(y_{1}, y_{2}\right) & \text { for } \quad\left(y_{1}, y_{2}\right) \in \partial S \\
\chi_{j}\left(y_{1},-y_{2}\right)=-\chi_{j}\left(y_{1}, y_{2}\right) & \text { for } \quad j \in\{2, \ldots, m\},\left(y_{1}, y_{2}\right) \in \partial S
\end{aligned}
$$




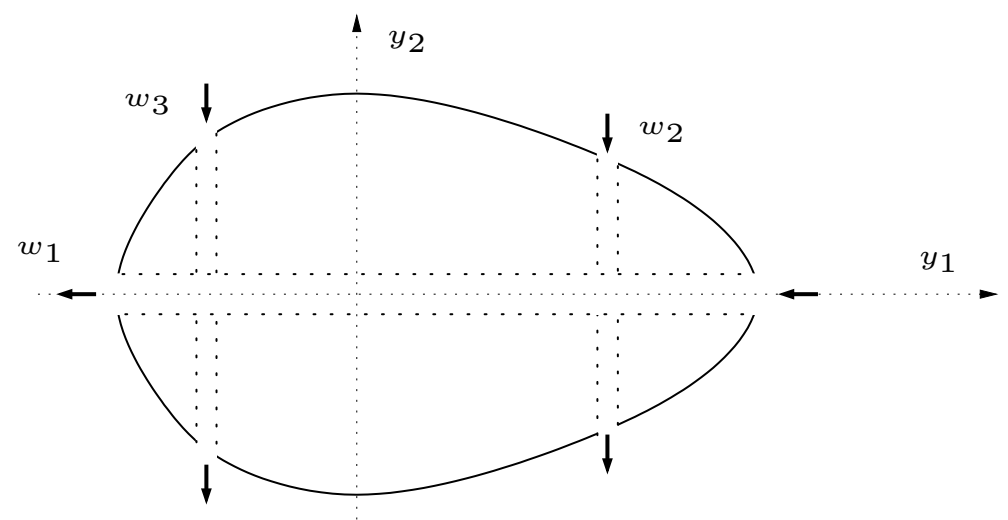

FiguRE 2. Three tunnel thrusters

Then the functions $(v, q, l, r)$ satisfy the following system

$$
\begin{aligned}
\frac{\partial v}{\partial t}+\left(v-l-r y^{\perp}\right) \cdot \nabla v+r v^{\perp}+\nabla q=0 & \text { in }[0, T] \times \Omega, \\
\operatorname{div} v=0 & \text { in }[0, T] \times \Omega, \\
v \cdot n=\left(l+r y^{\perp}\right) \cdot n+\sum_{1 \leqslant j \leqslant m} w_{j}(t) \chi_{j}(y) & \text { on }[0, T] \times \partial \Omega, \\
\lim _{|y| \rightarrow \infty} v(t, y)=0, & \text { in }[0, T], \\
m i=\int_{\partial \Omega} q n d \sigma-m r l^{\perp} & \text { in }[0, T], \\
J \dot{r}=\int_{\partial \Omega} q n \cdot y^{\perp} d \sigma & \text { in }[0, T], \\
v(0, y)=v_{0}(y) & \text { in } \Omega, \\
(l(0), r(0))=\left(l_{0}, r_{0}\right), &
\end{aligned}
$$

where $v_{0}(y)=Q\left(\theta_{0}\right)^{*} u_{0}\left(Q\left(\theta_{0}\right) y+h_{0}\right)$ and $l_{0}=Q\left(\theta_{0}\right)^{*} h_{1}$.

The paper is organized as follows.

In Section 2, we first consider potential flows. In that case, we obtain a finite-dimensional system similar to Kirchhoff laws, in which the control input $w$ appears through both linear terms (with time derivative) and bilinear terms. To investigate the controllability of such a system, we apply the return method due to Jean-Michel Coron (we refer the reader to [7] for an exposition of that method for finite-dimensional systems and for PDEs). We consider the linearization along a certain closed-loop trajectory and obtain a local controllability result (Theorem 2.9) assuming that two conditions are fulfilled, by using a variant of Silverman-Meadows test for the controllability of a time-varying linear system. A difficulty in the previous result is that the control may be different from 0 at the final time. This inconvenient disappears for certain values of the constants in the system, leading to a (global) steady-state controllability result (Theorem 2.14).

Next, we come back to the original system (1.18)-(1.25) in Section 3. We prove that it admits a global solution for a convenient choice of the vorticity at the inflow section of $\partial \Omega$ such that the difference between the present velocity and the potential velocity can be estimated by some measurement of the vorticity at time $t=0$ (Proposition 3.1). 
Combining the results in Sections 2 and 3, we obtain in Section 4 the main result of the paper (Theorem 4.1), namely a local controllability result for the dynamics of the boat even if the flow is not potential. Such a result, which may be seen as a "linearization" with respect to the vorticity, involves in its proof a topological argument (Lemma 4.2).

Finally Section 5 is an appendix containing some computations that can be skipped during a first reading.

\section{Potential Flows}

\subsection{Equations of the motion in the potential case.}

In this section we derive the equations describing the motion of the rigid body subject to flow controls on $\partial \Omega$ when the flow of the fluid is potential. We still denote by $v_{0}(\cdot)$ the velocity of the fluid at $t=0$, and here we assume that

$$
\begin{aligned}
\operatorname{curl} v_{0} & =0 \quad \text { in } \Omega \\
\int_{\partial \Omega} v_{0} \cdot \tau d \sigma & =0 .
\end{aligned}
$$

We also assume that the vorticity $\omega=\operatorname{curl} v$ is null at the inflow part of $\partial \Omega$, i.e.

$$
\omega(t, y)=0 \quad \text { if } \quad \sum_{j=1}^{m} w_{j}(t) \chi_{j}(y)<0 .
$$

Proposition 2.1. Under the assumptions (2.1), (2.2) and (2.3), one has

$$
\begin{gathered}
\omega=\operatorname{curl} v=0 \text { in }[0, T] \times \Omega, \\
\int_{\partial \Omega(t)} v(t, y) \cdot \tau d \sigma=0 \text { in }[0, T] .
\end{gathered}
$$

For the sake of completeness, Proposition 2.1 is proven in Section 5.

Now it follows from (1.19), (1.21), (2.4) and (2.5) that the flow is potential, i.e.

$$
v=\nabla \phi
$$

where $\phi=\phi(t, y)$ solves

$$
\begin{gathered}
\Delta \phi=0 \text { in }[0, T] \times \Omega, \\
\frac{\partial \phi}{\partial n}=\left(l+r y^{\perp}\right) \cdot n+\sum_{1 \leqslant j \leqslant m} w_{j}(t) \chi_{j}(y) \text { on }[0, T] \times \partial \Omega, \\
\lim _{|y| \rightarrow \infty} \nabla \phi(t, y)=0 \text { on }[0, T] .
\end{gathered}
$$

Actually, $\phi$ may be decomposed as

$$
\phi(t, y)=\sum_{1 \leqslant i \leqslant 2} l_{i}(t) \Phi_{i}(y)+r(t) \Phi_{3}(y)+\sum_{1 \leqslant j \leqslant m} w_{j}(t) \Psi_{j}(y)
$$

where $\Phi_{i}, i \in\{1,2,3\}$, solves

$$
\begin{gathered}
\Delta \Phi_{i}=0, \text { in } \Omega \\
\frac{\partial \Phi_{i}}{\partial n}(y)=K_{i}(y) \text { on } \partial \Omega \\
\lim _{|y| \rightarrow \infty} \nabla \Phi_{i}(y)=0,
\end{gathered}
$$


with

$$
K_{i}(y)=n(y) \cdot e_{i}=n_{i}(y) \text { for } i=1,2 \text { and } K_{3}(y)=y^{\perp} \cdot n(y),
$$

and where $\Psi_{j}, j=1, . ., m$ solve

$$
\begin{gathered}
\Delta \Psi_{j}=0 \text { in } \Omega, \\
\frac{\partial \Psi_{j}}{\partial n}=\chi_{j}(y) \text { on } \partial \Omega, \\
\lim _{|y| \rightarrow \infty} \nabla \Psi_{j}(y)=0 .
\end{gathered}
$$

Let $\widehat{H}^{s}(\Omega)$ denote the homogeneous Sobolev space

$$
\widehat{H}^{s}(\Omega)=\left\{q \in L_{l o c}^{2}(\bar{\Omega}) ; \nabla q \in H^{s-1}(\Omega)\right\},
$$

where $q \in L_{\text {loc }}^{2}(\bar{\Omega})$ means that $q \in L^{2}\left(\Omega \cap B_{0}\right)$ for any ball $B_{0} \subset \mathbb{R}^{2}$ with $B_{0} \cap \Omega \neq \emptyset$. Then we have the following result.

Lemma 2.2. Let $s \geqslant 1$ and $g \in H^{s-\frac{1}{2}}(\partial \Omega)$ be such that $\int_{\partial \Omega} g d \sigma=0$.

1. There exists a solution $\Psi \in \widehat{H}^{s+1}(\Omega)$ of the system

$$
\begin{gathered}
\Delta \Psi=0 \quad \text { in } \Omega, \\
\frac{\partial \Psi}{\partial n}=g \quad \text { on } \partial \Omega,
\end{gathered}
$$

and this solution is unique up to the addition of an arbitrary constant.

2. Assume that $s>1$. Then

$$
\limsup _{|y| \rightarrow \infty}\left(|y|^{2}|\nabla \Psi(y)|+|y|^{3}\left|\nabla^{2} \Psi(y)\right|\right)<\infty .
$$

and we may pick $\Psi$ so that $\lim _{|y| \rightarrow \infty} \Psi(y)=0$.

The proof of the first part of Lemma 2.2 may be done along the same lines as [20, Proposition 3.1]. For the second part, it is sufficient to write $\Psi\left(y_{1}, y_{2}\right)=\operatorname{Re} f\left(y_{1}+i y_{2}\right)$ where $f$ is some holomorphic function on $\Omega \subset \mathbb{R}^{2} \sim \mathbb{C}$ and to note that the expansion as a Laurent series of $f$ reads $f\left(y_{1}+i y_{2}\right)=\sum_{k \leqslant 0} a_{k}\left(y_{1}+i y_{2}\right)^{k}$ for some sequence $\left(a_{k}\right)_{k \leqslant 0}$ in $\mathbb{C}$, since $\lim _{|y| \rightarrow \infty} \nabla \Psi(y)=0$.

As the domain $S$ occupied by the rigid body and the functions $\chi_{j}, 1 \leqslant j \leqslant m$, supporting the control are assumed to be smooth, we infer that the functions $\nabla \Phi_{i}(i=1,2,3)$ and the functions $\nabla \Psi_{j}(1 \leqslant j \leqslant m)$ are in the space $H^{\infty}(\Omega)$. As a consequence, we notice that for all $i=1,2,3$ and $j=1, \ldots, m$,

$$
\begin{aligned}
\int_{\partial \Omega} \Phi_{i} K_{j} d \sigma & =\int_{\Omega} \nabla \Phi_{i} \cdot \nabla \Phi_{j} d y=\int_{\partial \Omega} K_{i} \Phi_{j} d \sigma, \\
\int_{\partial \Omega} \Psi_{i} K_{j} d \sigma & =\int_{\Omega} \nabla \Psi_{i} \cdot \nabla \Phi_{j} d y=\int_{\partial \Omega} \chi_{i} \Phi_{j} d \sigma .
\end{aligned}
$$

Let us now reformulate the equations for the motion of the rigid body. We define the matrix $\mathcal{M} \in \mathbb{R}^{3 \times 3}$ by

$$
\mathcal{M}_{i j}=\int_{\Omega} \nabla \Phi_{i} \cdot \nabla \Phi_{j} d y,
$$

and the (added mass) inertia matrix $\mathcal{J}$ by

$$
\mathcal{J}=\left[\begin{array}{cc}
\operatorname{mId}_{2} & 0 \\
0 & J
\end{array}\right]+\mathcal{M}
$$

Since $\mathcal{M}$ is a Gram matrix, it is nonnegative. It follows that $\mathcal{J}$ is a positive definite matrix, hence it is invertible. 
Remark 2.3. Under the assumptions (1.15) and $m=3$, one easily infer from Proposition 5.2 (see below) that $\mathcal{M}$ takes the following form

$$
\mathcal{M}=\left(\begin{array}{ccc}
\int_{\partial \Omega} \Phi_{1} n_{1} d \sigma & 0 & 0 \\
0 & \int_{\partial \Omega} \Phi_{2} n_{2} d \sigma & \int_{\partial \Omega} \Phi_{2} n \cdot y^{\perp} d \sigma \\
0 & \int_{\partial \Omega} \Phi_{2} n \cdot y^{\perp} d \sigma & \int_{\partial \Omega} \Phi_{3} n \cdot y^{\perp} d \sigma
\end{array}\right)
$$

If, in addition,

$$
\left(y_{1}, y_{2}\right) \in S \Rightarrow\left(-y_{1}, y_{2}\right) \in S
$$

then both $\mathcal{M}$ and $\mathcal{J}$ are diagonal.

Let us set

$$
q=\left(q_{1}, q_{2}, q_{3}\right)=\left(h_{1}, h_{2}, \theta\right), p=\left(p_{1}, p_{2}, p_{3}\right)=\left(l_{1}, l_{2}, r\right) \text { and } w=\left(w_{1}, \ldots, w_{m}\right) .
$$

Recall that $\dot{h}=Q(\theta) l$. Introduce the $3 \times 3$ matrix

$$
\mathcal{Q}(q)=\left(\begin{array}{cc}
Q\left(q_{3}\right) & 0 \\
0 & 1
\end{array}\right)
$$

In the potential case, the dynamics of the boat can be written in the following way.

Proposition 2.4. The dynamics of $(q, p)$ read

$$
\begin{aligned}
& \dot{q}=\mathcal{Q}(q) p, \\
& \dot{p}=\mathcal{J}^{-1}(C \dot{w}+F(p, w)),
\end{aligned}
$$

where $C$ denotes the constant matrix

$$
\left(\begin{array}{cccc}
c_{1} & 0 & \cdots & 0 \\
0 & c_{2} & \cdots & c_{m} \\
0 & \tilde{c}_{2} & \cdots & \tilde{c}_{m}
\end{array}\right):=\left(\begin{array}{cccc}
-\int_{\partial \Omega} \Psi_{1} K_{1} d \sigma & 0 & \cdots & 0 \\
0 & -\int_{\partial \Omega} \Psi_{2} K_{2} d \sigma & \cdots & -\int_{\partial \Omega} \Psi_{m} K_{2} d \sigma \\
0 & -\int_{\partial \Omega} \Psi_{2} K_{3} d \sigma & \cdots & -\int_{\partial \Omega} \Psi_{m} K_{3} d \sigma
\end{array}\right)
$$

and $F(p, w)$ is composed of bilinear terms in $p_{i}, 1 \leqslant i \leqslant 3$, and $w_{j}, 1 \leqslant j \leqslant m$.

The detailed computations yielding Proposition 2.4 are given in Appendix. In particular, the equation (2.26) with the term $F(p, w)$ is made explicit in (5.32) -(5.34).

\subsection{Elementary approaches.}

Let us have a look at the linearization of (2.25)-(2.26) at the origin, namely

$$
\begin{aligned}
\dot{q} & =p, \\
\dot{p} & =\mathcal{J}^{-1} C \dot{w} .
\end{aligned}
$$

The following result relates the controllability of (2.27)-(2.28) to the rank of $C$.

Proposition 2.5. The linearized system (2.27)-(2.28) with control input $\dot{w} \in \mathbb{R}^{m}$ is controllable if and only if rank $(C)=3$.

Proof. To apply Kalman rank test (see e.g. [7, 22]), we compute

$$
\operatorname{rank}\left(\left(\begin{array}{c}
0 \\
\mathcal{J}^{-1} C
\end{array}\right),\left(\begin{array}{cc}
0 & I \\
0 & 0
\end{array}\right)\left(\begin{array}{c}
0 \\
\mathcal{J}^{-1} C
\end{array}\right)\right)=2 \operatorname{rank}(C)
$$

and the result follows at once.

In particular, the controllability of the linearized system requires that the number $m$ of control inputs satisfies $m \geqslant 3$. 
Example 2.6. When the boat has an elliptic shape, i.e.

$$
S=\left\{y \in \mathbb{R}^{2} ; \frac{y_{1}^{2}}{a^{2}}+\frac{y_{2}^{2}}{b^{2}}<1\right\},
$$

where $a>b>0$ are some given constants, then the functions $\Phi_{i}, i=1,2,3$, may be explicitly computed by using complex analysis (see [4]). Letting $y_{\theta}=(a \cos \theta, b \sin \theta)$ for $-\pi \leqslant \theta \leqslant \pi$, we easily obtain that

$$
\Phi_{1}\left(y_{\theta}\right)=-b \cos \theta, \quad \Phi_{2}\left(y_{\theta}\right)=-a \sin \theta, \quad \text { and } \Phi_{3}\left(y_{\theta}\right)=\frac{b^{2}-a^{2}}{4} \sin (2 \theta),
$$

Assuming that $m=3$ and letting $d \mu=\left|y_{\theta}^{\prime}\right| d \theta$, we arrive to

$$
\begin{aligned}
C & =\left(\begin{array}{ccc}
-\int_{\partial \Omega} \Phi_{1} \chi_{1} d \sigma & 0 & 0 \\
0 & -\int_{\partial \Omega} \Phi_{2} \chi_{2} d \sigma & -\int_{\partial \Omega} \Phi_{2} \chi_{3} d \sigma \\
0 & -\int_{\partial \Omega} \Phi_{3} \chi_{2} d \sigma & -\int_{\partial \Omega} \Phi_{3} \chi_{3} d \sigma
\end{array}\right) \\
& =\left(\begin{array}{ccc}
b \int_{0}^{2 \pi} \chi_{1}\left(y_{\theta}\right) \cos \theta d \mu & 0 & 0 \\
0 & a \int_{0}^{2 \pi} \chi_{2}\left(y_{\theta}\right) \sin \theta d \mu & a \int_{0}^{2 \pi} \chi_{3}\left(y_{\theta}\right) \sin \theta d \mu \\
0 & \frac{a^{2}-b^{2}}{4} \int_{0}^{2 \pi} \chi_{2}\left(y_{\theta}\right) \sin (2 \theta) d \mu & \frac{a^{2}-b^{2}}{4} \int_{0}^{2 \pi} \chi_{3}\left(y_{\theta}\right) \sin (2 \theta) d \mu
\end{array}\right) .
\end{aligned}
$$

Assuming

$$
\begin{array}{r}
\chi_{1}\left(y_{\theta}\right) \cos \theta \geqslant 0, \quad \chi_{2}\left(y_{\theta}\right) \sin \theta \geqslant 0, \quad \chi_{3}\left(y_{\theta}\right) \sin \theta \leqslant 0, \\
\text { Supp } \chi_{2} \subset\{-\pi / 2<\theta<\pi / 2\} \quad \text { and } \operatorname{Supp} \chi_{3} \subset\{\pi / 2<|\theta|<\pi\}
\end{array}
$$

we infer that $c_{1}, c_{2},-c_{3}, \tilde{c}_{2}$ and $\tilde{c}_{3}$ are all (strictly) positive. This yields that $\operatorname{det} C>0$, hence it follows from Proposition 2.5 that system (2.27)-(2.28) is controllable.

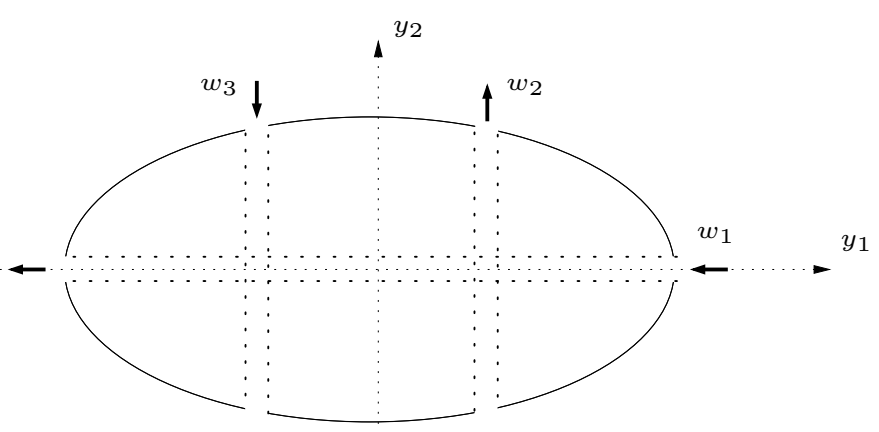

FiguRE 3. Elliptic boat with three controls

From now on we assume that $m=2$.

Before investigating the controllability of the full system (2.25)-(2.26), let us consider first the case when $w_{2} \equiv 0$ and $q_{2}(0)=q_{3}(0)=p_{2}(0)=p_{3}(0)=0$. Then, in view of 
(5.32)-(5.34), $q_{2}=p_{2}=q_{3}=p_{3} \equiv 0$, and (2.25)-(2.26) reduces to the system

$$
\begin{aligned}
\dot{q}_{1} & =p_{1}, \\
\dot{p}_{1} & =-\left(m+\int_{\partial \Omega} \Phi_{1} n_{1}\right)^{-1}\left(\dot{w}_{1} \int_{\partial \Omega} \Psi_{1} n_{1}+w_{1} p_{1} \int_{\partial \Omega} \chi_{1} \partial_{1} \Phi_{1}+w_{1}^{2} \int_{\partial \Omega} \chi_{1} \partial_{1} \Psi_{1}\right) \\
& =: \alpha \dot{w}_{1}+\beta w_{1} p_{1}+\gamma w_{1}^{2} .
\end{aligned}
$$

In the rest of the paper, we suppose that $\chi_{1}$ is chosen so that

$$
\alpha \neq 0 \text {. }
$$

A natural idea would be to transform (2.30)-(2.31) into a system with $\left(q_{1}, p_{1}, w_{1}\right)$ as state and $\rho_{1}:=\dot{w}_{1}$ as control, namely

$$
\begin{aligned}
\dot{q}_{1} & =p_{1}, \\
\dot{p}_{1} & =\alpha \rho_{1}+\beta w_{1} p_{1}+\gamma w_{1}^{2}, \\
\dot{w}_{1} & =\rho_{1} .
\end{aligned}
$$

Unfortunately, this approach does not work, as it is shown by the following result.

Lemma 2.7. System (2.33)-(2.35) fails to be (locally or globally) controllable.

Proof. To prove Lemma 2.7, we consider the function $z_{1}:=p_{1}-\alpha w_{1}$. We notice that

$$
\dot{z}_{1}=\beta w_{1} z_{1}+(\gamma+\alpha \beta) w_{1}^{2},
$$

hence

$$
z_{1}(t)=\left(z_{1}(0)+(\gamma+\alpha \beta) \int_{0}^{t} w_{1}^{2}(\tau) e^{-\int_{0}^{\tau} \beta w_{1}(s) d s} d \tau\right) e^{\int_{0}^{t} \beta w_{1}(s) d s} .
$$

Therefore, if $z_{1}(0)=0$, then $z_{1} \equiv 0$ if $\gamma+\alpha \beta=0$, and $z_{1}$ has the same sign as $\gamma+\alpha \beta$ otherwise. It follows that (2.33)-(2.35) fails to be (locally or globally) controllable, and the lemma is proved.

Thus we cannot control both $\left(q_{1}, p_{1}\right)$ and $w_{1}$. One may wonder whether it is possible to require that the fluid be at rest when the rigid body is, that is if the condition

$$
w_{1}(0)=w_{1}(T)=0
$$

may be imposed when $p_{1}(0)=p_{1}(T)=0$. The following result shows that this occurs for a very particular set of coefficients.

Lemma 2.8. Let $T>0$. Then we may associate to each pair $\left(q_{1}^{0}, q_{1}^{T}\right)$ in $\mathbb{R}^{2}$ a control input $w_{1} \in H_{0}^{1}(0, T)$ such that the solution $\left(q_{1}(t), p_{1}(t)\right)$ of $(2.30)-(2.31)$ emanating from $\left(q_{1}^{0}, 0\right)$ at $t=0$ reaches $\left(q_{1}^{T}, 0\right)$ at $t=T$ if and only if

$$
\gamma+\alpha \beta=0 \text {. }
$$

Proof. Assume first that (2.38) holds, and that $p_{1}(0)=0$. Pick any pair $\left(q_{1}^{0}, q_{1}^{T}\right)$ in $\mathbb{R}^{2}$ and consider again the function $z_{1}(t)=p_{1}(t)-\alpha w_{1}(t)$. If $w_{1}(0)=0$, then $z_{1}(0)=0$ and from (2.38) we infer that

$$
p_{1}-\alpha w_{1}=z_{1} \equiv 0 .
$$

As it is well known, the control system $\dot{q}_{1}=\alpha w_{1}$ may be controlled by using control inputs in $C_{0}^{\infty}(0, T)$. Conversely, $(2.38)$ is a necessary condition, as $(2.36)$ with $z_{1}(0)=z_{1}(T)=0$ and $w_{1} \not \equiv 0$ yields $\gamma+\alpha \beta=0$. 


\subsection{Local controllability of the potential model.}

Thereafter, we will still assume that $\left(w_{1}(0), w_{2}(0)\right)=(0,0)$, but we shall refrain from imposing the condition $\left(w_{1}(T), w_{2}(T)\right)=(0,0)$. The main result in this section (see below Theorem 2.9) is derived in following a strategy inspired in part from Coron's return method. It is organized as follows:

- Step 1. We construct a (non trivial) trajectory $(\bar{q}, \bar{p}, \bar{w})$ such that

$$
(\bar{q}(0), \bar{p}(0))=(\bar{q}(T), \bar{p}(T))=(0,0) .
$$

To do that, proceeding as in the flatness approach by Fliess et al. [8], we consider a particular trajectory $\left(\bar{q}_{1}, \bar{p}_{1}\right)$ of $(2.30)$ and next define the control input $\bar{w}_{1}$ by solving the Cauchy problem (2.31) together with the initial condition $\bar{w}_{1}(0)=0$. Notice that $\bar{w}_{1}(T)$ may be different from 0 , and that $\bar{w}_{1}$ is not required to be odd with respect to the time $T / 2$. On the other hand, $\bar{w}_{2} \equiv 0$ so that $\bar{q}_{2}=\bar{p}_{2}=\bar{q}_{3}=$ $\bar{p}_{3} \equiv 0$.

- Step 2. We show that the linearization along this trajectory is controllable (under suitable assumptions) by combining the classical Silverman-Meadows criterion to a test for the controllability of a linear system involving a control input together with its time derivative.

- Step 3. The local controllability of the nonlinear system (2.25)-(2.26) is then established with the aid of a linearization argument.

For notational convenience, we introduce the matrices

$$
\mathbf{A}=\left(\begin{array}{lll}
a_{1} & 0 & 0 \\
0 & a_{2} & a_{3}+\alpha a_{4} \\
0 & a_{5}+\alpha a_{6} & a_{7}+\alpha a_{8}
\end{array}\right) \quad \text { and } \mathbf{B}=\left(\begin{array}{ll}
b_{1}+\alpha b_{2} & 0 \\
0 & b_{3}+\alpha b_{4} \\
0 & b_{5}+\alpha b_{6}
\end{array}\right)
$$

where

$$
\begin{gathered}
a_{1}=-\int_{\partial \Omega} \chi_{1} \partial_{1} \Phi_{1} d \sigma, \quad a_{2}=-\int_{\partial \Omega} \chi_{1} \partial_{2} \Phi_{2} d \sigma, \quad a_{3}=-\int_{\partial \Omega} y_{2} \nabla \Psi_{1} \cdot \tau d \sigma-\int_{\partial \Omega} \chi_{1} \partial_{2} \Phi_{3} d \sigma, \\
a_{4}=-m-\int_{\partial \Omega} y_{2} \nabla \Phi_{1} \cdot \tau d \sigma, \quad a_{5}=\int_{\partial \Omega} y_{2} \nabla \Psi_{1} \cdot \tau d \sigma-\int_{\partial \Omega} \chi_{1} \nabla \Phi_{2} \cdot y^{\perp} d \sigma, \\
a_{6}=\int_{\partial \Omega} y_{1} \nabla \Phi_{2} \cdot \tau d \sigma+\int_{\partial \Omega} y_{2} \nabla \Phi_{1} \cdot \tau d \sigma, \quad a_{7}=-\int_{\partial \Omega} \chi_{1} \nabla \Phi_{3} \cdot y^{\perp} d \sigma, \quad a_{8}=\int_{\partial \Omega} y_{1} \nabla \Phi_{3} \cdot n d \sigma,
\end{gathered}
$$

and

$$
\begin{gathered}
b_{1}=-2 \int_{\partial \Omega} \chi_{1} \partial_{1} \Psi_{1} d \sigma, \quad b_{2}=-\int_{\partial \Omega} \chi_{1} \partial_{1} \Phi_{1} d \sigma, \quad b_{3}=-\int_{\partial \Omega} \chi_{1} \partial_{2} \Psi_{2} d \sigma-\int_{\partial \Omega} \chi_{2} \partial_{2} \Psi_{1} d \sigma, \\
b_{4}=-\int_{\partial \Omega} \chi_{2} \partial_{2} \Phi_{1} d \sigma, \quad b_{5}=-\int_{\partial \Omega} \chi_{1} \nabla \Psi_{2} \cdot y^{\perp} d \sigma-\int_{\partial \Omega} \chi_{2} \nabla \Psi_{1} \cdot y^{\perp} d \sigma, \\
b_{6}=\int_{\partial \Omega} y_{1} \nabla \Psi_{2} \cdot \tau d \sigma-\int_{\partial \Omega} \chi_{2} \nabla \Phi_{1} \cdot y^{\perp} d \sigma .
\end{gathered}
$$

Simple but tedious computations give

$$
b_{3}=-\int_{\partial \Omega}\left(\nabla \Psi_{1} \cdot \nabla \Psi_{2}\right) n_{2} d \sigma \quad \text { and } \quad b_{5}=-\int_{\partial \Omega}\left(\nabla \Psi_{1} \cdot \nabla \Psi_{2}\right)\left(n \cdot y^{\perp}\right) d \sigma .
$$

We also need to introduce the matrix

$$
\mathbf{D}=\left(\begin{array}{lll}
0 & 0 & 0 \\
0 & 0 & \alpha \\
0 & 0 & 0
\end{array}\right)
$$


The following result shows that, under suitable assumptions, the local controllability holds with only two control inputs.

Theorem 2.9. If both rank conditions

$$
\begin{aligned}
& \operatorname{rank}\left(C, \mathbf{B}+\mathbf{A} \mathcal{J}^{-1} C\right)=3, \\
& \operatorname{rank}\left(C, \frac{1}{2} \mathcal{J} \mathbf{D} \mathcal{J}^{-1} C+\mathbf{B}+\mathbf{A} \mathcal{J}^{-1} C\right)=3,
\end{aligned}
$$

are fulfilled, then for any $T>0$ the system

$$
\begin{aligned}
\dot{q} & =\mathcal{Q}(q) p, \\
\mathcal{J} \dot{p} & =C \dot{w}+F(p, w),
\end{aligned}
$$

with state $(q, p) \in \mathbb{R}^{6}$ and control $w \in \mathbb{R}^{2}$ is locally controllable around the origin in time $T$. We can also impose that the control input $w$ satisfies $w(0)=0$. Moreover, for some $\eta>0$, there is a $C^{1}$ map from $B_{\mathbb{R}^{12}}(0, \eta)$ to $H^{2}\left(0, T, \mathbb{R}^{2}\right)$, which associates to $\left(q_{0}, p_{0}, q_{T}, p_{T}\right)$ a control satisfying $w(0)=0$ and steering the state of the system from $\left(q_{0}, p_{0}\right)$ at $t=0$ to $\left(q_{T}, p_{T}\right)$ at $t=T$.

Remark 2.10. (1) In the limit $m \rightarrow \infty$ and $J \rightarrow \infty,(2.42)$ and (2.43) become $\left|c_{1}\right|+$ $\left|b_{1}\right|>0$ and $\operatorname{rank}\left[\begin{array}{cc}c_{2} & b_{3} \\ \tilde{c}_{2} & b_{5}\end{array}\right]=2$.

(2) The condition $w(0)=0$ means that the boundary condition for the fluid velocity at $t=0$ reduces to $v \cdot n=\left(l+r y^{\perp}\right) \cdot n$.

(3) If instead of (2.42) the slightly stronger assumption rank $\left(\left(c_{1} 00\right)^{*}, \mathbf{B}+\mathbf{A} \mathcal{J}^{-1} C\right)=$ 3 holds, then we can impose that $w_{1}(0)=w_{2}(0)=w_{2}(T)=0$. Nevertheless, it seems that $w_{1}(T) \neq 0$ in general, unless $\gamma+\alpha \beta=0$ (see Theorem 2.14 below).

Example 2.11. Let us consider again a boat with an elliptic shape, which is equipped with two tunnel thrusters parallel to the $y_{1}$-axis (see Figure 4).

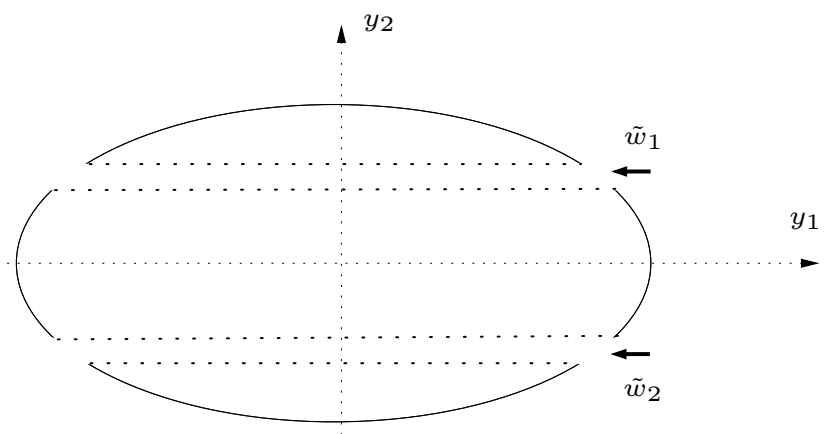

FiguRE 4. Elliptic shape with two tunnel thrusters

Assume that the normal component of the velocity is controlled as follows

$$
v \cdot n=\left(l+r y^{\perp}\right) \cdot n+\tilde{w}_{1}(t) \chi(y)+\tilde{w}_{2}(t) \chi\left(y^{\prime}\right)
$$

where $y^{\prime}=\left(y_{1},-y_{2}\right)$, Supp $\chi \subset\left\{y_{2}>0\right\}$, and $\chi\left(-y_{1}, y_{2}\right)=-\chi\left(y_{1}, y_{2}\right)$ for $y \in \partial S$. Introducing $\chi_{1}(y)=\left(\chi(y)+\chi\left(y^{\prime}\right)\right) / 2$ and $\chi_{2}(y)=\left(\chi(y)-\chi\left(y^{\prime}\right)\right) / 2$, we note that $(1.16)$ and (1.17) are satisfied, and that (1.20) holds with $m=2$ and $w_{1}:=\tilde{w}_{1}+\tilde{w}_{2}, w_{2}:=\tilde{w}_{1}-\tilde{w}_{2}$. It 
is easy to see that $c_{1} \neq 0, \alpha \neq 0, c_{2}=-\int_{\partial \Omega} \chi_{2} \Phi_{2} d \sigma=0$, and that $\tilde{c}_{2}=-\int_{\partial \Omega} \chi_{2} \Phi_{3} d \sigma \neq 0$. Thus the rank condition in Remark 2.10 (i) is satisfied if and only if

$$
b_{3}=-\int_{\partial \Omega}\left(\nabla \Psi_{1} \cdot \nabla \Psi_{2}\right) n_{2} d \sigma=-\frac{1}{2} \int_{\partial \Omega}|\nabla \Psi|^{2} n_{2} d \sigma \neq 0,
$$

where $\Psi$ solves (2.17)-(2.18) with $g=\chi$.

The rest of Paragraph 2.3 is devoted to the proof of Theorem 2.9. We divide the proof according to the three steps described above.

2.3.1. Step 1. Construction of a loop-shaped trajectory. Pick any $\lambda_{0}>0$ and let $\lambda \in$ $\left(0, \lambda_{0}\right)$. Set

$$
\bar{q}_{1}(t)=\lambda(1-\cos (2 \pi t / T)), \quad \bar{p}_{1}(t)=\lambda(2 \pi / T) \sin (2 \pi t / T)
$$

and define $\bar{w}_{1}$ as the solution to the Cauchy problem

$$
\begin{aligned}
\dot{\bar{w}}_{1} & =\alpha^{-1}\left(\dot{\bar{p}}_{1}-\beta w_{1} p_{1}-\gamma w_{1}^{2}\right) \\
\bar{w}_{1}(0) & =0
\end{aligned}
$$

By a classical result on the continuous dependence of solutions of ODE with respect to a parameter, we see that the solution $w_{1}$ of $(2.46)-(2.47)$ is defined on $[0, T]$ provided that $\lambda_{0}$ is small enough. We "complete" $\left(\bar{q}_{1}, \bar{p}_{1}, \bar{w}_{1}\right)$ into a solution $(\bar{q}, \bar{p}, \bar{w})$ of $(2.25)-(2.26)$ be letting $\bar{q}_{i}=\bar{p}_{i}=0$ for $i=2,3$ and $\bar{w}_{2} \equiv 0$. Then $(\bar{q}, \bar{p})(0)=(0,0)=(\bar{q}, \bar{p})(T)$.

2.3.2. Step 2. Controllability of the linearized system. Writing $q=\bar{q}+z, p=\bar{p}+k$, $w=\bar{w}+f$, expanding in (2.25)-(2.26), and keeping only the first order terms in $z, k$, and $f$, we obtain the following linear system

$$
\begin{aligned}
\left(\begin{array}{c}
\dot{z} \\
\dot{k}
\end{array}\right) & =\left(\begin{array}{cc}
D(t) & I \\
0 & \mathcal{J}^{-1} A(t)
\end{array}\right)\left(\begin{array}{l}
z \\
k
\end{array}\right)+\left(\begin{array}{c}
0 \\
\mathcal{J}^{-1} B(t)
\end{array}\right)\left(\begin{array}{c}
f_{1} \\
f_{2}
\end{array}\right)+\left(\begin{array}{c}
0 \\
\mathcal{J}^{-1} C
\end{array}\right)\left(\begin{array}{l}
\dot{f}_{1} \\
\dot{f}_{2}
\end{array}\right) \\
& =: \mathcal{A}(t)\left(\begin{array}{c}
z \\
k
\end{array}\right)+\mathcal{B}(t)\left(\begin{array}{l}
f_{1} \\
f_{2}
\end{array}\right)+\mathcal{C}\left(\begin{array}{l}
\dot{f}_{1} \\
\dot{f}_{2}
\end{array}\right)
\end{aligned}
$$

where

$$
\begin{aligned}
& A(t)=\left(\begin{array}{lll}
a_{1} \bar{w}_{1} & 0 & 0 \\
0 & a_{2} \bar{w}_{1} & a_{3} \bar{w}_{1}+a_{4} \bar{p}_{1} \\
0 & a_{5} \bar{w}_{1}+a_{6} \bar{p}_{1} & a_{7} \bar{w}_{1}+a_{8} \bar{p}_{1}
\end{array}\right), \\
& B(t)=\left(\begin{array}{ll}
b_{1} \bar{w}_{1}+b_{2} \bar{p}_{1} & 0 \\
0 & b_{3} \bar{w}_{1}+b_{4} \bar{p}_{1} \\
0 & b_{5} \bar{w}_{1}+b_{6} \bar{p}_{1}
\end{array}\right), \\
& \text { and } \quad D(t)=\left(\begin{array}{ccc}
0 & 0 & 0 \\
0 & 0 & \bar{p}_{1} \\
0 & 0 & 0
\end{array}\right) \text {. }
\end{aligned}
$$

For a time-varying linear system

$$
\dot{x}=\mathcal{A}(t) x+\mathcal{B}(t) u, \quad x \in \mathbb{R}^{n}, u \in \mathbb{R}^{m}
$$

we denote by $\mathcal{R}_{T}(\mathcal{A}, \mathcal{B})$ the reachable space in time $T$ from the origin, i.e.

$$
\mathcal{R}_{T}(\mathcal{A}, \mathcal{B})=\left\{x_{T} \in \mathbb{R}^{n}\right.
$$

$\exists u \in L^{2}\left(0, T ; \mathbb{R}^{m}\right), x_{T}=x(T)$ where $x(\cdot)$ solves $(2.49)$ and $\left.x(0)=0\right\}$. 
If $\phi(t, s) \in \mathbb{R}^{n \times n}$ denotes the fundamental solution associated with $\mathcal{A}(\cdot)$, i.e. the solution of the Cauchy problem

$$
\begin{gathered}
\frac{\partial \phi}{\partial t}=\mathcal{A}(t) \phi(t, s), \\
\phi(s, s)=I,
\end{gathered}
$$

then

$$
\mathcal{R}_{T}(\mathcal{A}, \mathcal{B})=\left\{\int_{0}^{T} \phi(T, t) \mathcal{B}(t) u(t) d t ; u \in L^{2}\left(0, T ; \mathbb{R}^{m}\right)\right\} .
$$

We shall apply a controllability test due to Silverman-Meadows (see [7]) in a slightly extended form. For the sake of completeness, we prove it in the appendix.

Define a sequence of matrices $\mathcal{M}_{i}(t) \in \mathbb{R}^{n \times m}$ by

$$
\mathcal{M}_{0}(t)=\mathcal{B}(t) \text { and } \mathcal{M}_{i}(t)=\dot{\mathcal{M}}_{i-1}(t)-\mathcal{A}(t) \mathcal{M}_{i-1}(t) \quad \forall i \geqslant 1, \forall t \in[0, T] .
$$

Proposition 2.12. Let $\varepsilon>0, \mathcal{A} \in C^{\omega}\left((-\varepsilon, T+\varepsilon) ; \mathbb{R}^{n \times n}\right)$ and $\mathcal{B} \in C^{\omega}\left((-\varepsilon, T+\varepsilon) ; \mathbb{R}^{n \times m}\right)$, and let $\left(\mathcal{M}_{i}\right)_{i \geqslant 0}$ be the sequence defined in (2.53). Then for all $t_{0} \in[0, T]$, we have

$$
\mathcal{R}_{T}(\mathcal{A}, \mathcal{B})=\operatorname{Span}\left\{\phi\left(T, t_{0}\right) \mathcal{M}_{i}\left(t_{0}\right) u ; u \in \mathbb{R}^{m}, i \geqslant 0\right\},
$$

where $\phi$ denotes the fundamental solution defined in (2.50)-(2.51).

Consider now the system

$$
\dot{x}=\mathcal{A}(t) x+\mathcal{B}(t) u+\mathcal{C} \dot{u},
$$

and denote by $\mathcal{R}$ the reachable set from the origin, i.e.

$$
\mathcal{R}=\left\{x_{T} \in \mathbb{R}^{n} ; \exists u \in H^{1}\left(0, T ; \mathbb{R}^{m}\right), x_{T}=x(T) \text { where } x(\cdot) \text { solves }(2.55) \text { and } x(0)=0\right\} .
$$

Note that $u$ may take arbitrary values at $t=0, T$. It may be necessary to impose that $u(0)=0$ and/or that $u(T)=0$. Accordingly, we introduce the spaces

$$
\begin{aligned}
\mathcal{R}_{u(0)=0} & =\left\{x_{T} \in \mathcal{R} \text { associated with some } u \in H^{1}\left(0, T ; \mathbb{R}^{m}\right) \text { with } u(0)=0\right\}, \\
\mathcal{R}_{u(0)=u(T)=0} & =\left\{x_{T} \in \mathcal{R} \text { associated with some } u \in H_{0}^{1}\left(0, T ; \mathbb{R}^{m}\right)\right\} .
\end{aligned}
$$

Note that, by an obvious density argument, we may as well assume that $u \in C^{\infty}\left([0, T], \mathbb{R}^{2}\right)$ in the above definitions of the reachable spaces, without changing these spaces.

Then the following result holds true.

Proposition 2.13. The reachable sets from the origin for the system (2.55) are respectively

$$
\begin{aligned}
\mathcal{R} & =\mathcal{R}_{T}(\mathcal{A}, \mathcal{B}+\mathcal{A C})+\mathcal{C} \mathbb{R}^{m}+\phi(T, 0) \mathcal{C} \mathbb{R}^{m} \\
\mathcal{R}_{u(0)=0} & =\mathcal{R}_{T}(\mathcal{A}, \mathcal{B}+\mathcal{A C})+\mathcal{C} \mathbb{R}^{m} \\
\mathcal{R}_{u(0)=u(T)=0} & =\mathcal{R}_{T}(\mathcal{A}, \mathcal{B}+\mathcal{A C})
\end{aligned}
$$

Proof. Any element of $\mathcal{R}$ takes the form $x=\int_{0}^{T} \phi(T, t)(\mathcal{B}(t) u(t)+\mathcal{C} \dot{u}(t)) d t$ for some $u \in$ $H^{1}\left(0, T ; \mathbb{R}^{m}\right)$. Integrating by part, and using the fact that $\partial[\phi(T, t)] / \partial t=-\phi(T, t) \mathcal{A}(t)$, we obtain

$$
x=\int_{0}^{T} \phi(T, t)(\mathcal{B}(t)+\mathcal{A}(t) \mathcal{C}) u(t) d t+\mathcal{C} u(T)-\phi(T, 0) \mathcal{C} u(0),
$$

hence

$$
\mathcal{R} \subset \mathcal{R}_{T}(\mathcal{A}, \mathcal{B}+\mathcal{A C})+\mathcal{C} \mathbb{R}^{m}+\phi(T, 0) \mathcal{C} \mathbb{R}^{m}
$$


As we may assume in (2.52) that $u$ ranges over $C_{0}^{\infty}\left(0, T, \mathbb{R}^{m}\right)$ (hence $u(0)=u(T)=0$ ), we infer that

$$
\mathcal{R}_{T}(\mathcal{A}, \mathcal{B}+\mathcal{A C}) \subset \mathcal{R} .
$$

On the other hand, taking $u(t)=t u_{0}\left(\right.$ resp. $\left.u(t)=(T-t) u_{0}\right)$ where $u_{0}$ is an arbitrary constant vector, and using (2.59), we obtain that $\mathcal{C} \mathbb{R}^{m} \subset \mathcal{R}$ (resp. $\phi(T, 0) \mathcal{C} \mathbb{R}^{m} \subset \mathcal{R}$ ). This completes the proof of (2.56). (2.57) and (2.58) follow at once.

We shall establish the controllability of (2.48) by combining Propositions 2.12 and 2.13. We denote by $\left(\mathcal{M}_{i}\right)_{i \geqslant 0}$ the sequence of matrices associated with the pair $(\mathcal{A}, \mathcal{B}+\mathcal{A C})$ (hence, $\left.\mathcal{M}_{0}(t)=\mathcal{B}(t)+\mathcal{A}(t) \mathcal{C}\right)$.

Straightforward but tedious computations give

$$
\begin{aligned}
\mathcal{M}_{0}(t)= & \left(\begin{array}{c}
\mathcal{J}^{-1} C \\
\mathcal{J}^{-1}\left(B(t)+A(t) \mathcal{J}^{-1} C\right)
\end{array}\right), \\
\mathcal{M}_{1}(t)= & \left(\begin{array}{c}
-D \mathcal{J}^{-1} C-\mathcal{J}^{-1}\left(B(t)+A(t) \mathcal{J}^{-1} C\right) \\
\mathcal{J}^{-1}\left[\dot{B}(t)+\dot{A}(t) \mathcal{J}^{-1} C-A(t) \mathcal{J}^{-1}\left(B(t)+A(t) \mathcal{J}^{-1} C\right)\right]
\end{array}\right), \\
\mathcal{M}_{2}(t)= & \left(\begin{array}{c}
-\dot{D} \mathcal{J}^{-1} C+D\left[D \mathcal{J}^{-1} C+\mathcal{J}^{-1}\left(B(t)+A(t) \mathcal{J}^{-1} C\right)\right] \\
-\mathcal{J}^{-1}\left[2\left(\dot{B}(t)+\dot{A}(t) \mathcal{J}^{-1} C\right)-A(t) \mathcal{J}^{-1}\left(B(t)+A(t) \mathcal{J}^{-1} C\right)\right] \\
\mathcal{J}^{-1}\left\{\ddot{B}(t)+\ddot{A}(t) \mathcal{J}^{-1} C-\frac{d}{d t}\left[A \mathcal{J}^{-1}\left(B+A \mathcal{J}^{-1} C\right)\right](t)\right. \\
\left.-A(t) \mathcal{J}^{-1}\left[\dot{B}(t)+\dot{A}(t) \mathcal{J}^{-1} C-A(t) \mathcal{J}^{-1}\left(B(t)+A(t) \mathcal{J}^{-1} C\right)\right]\right\}
\end{array}\right) .
\end{aligned}
$$

From Propositions 2.12 and 2.13, we know that

$$
\mathcal{R}_{f(0)=0}=\mathcal{C} \mathbb{R}^{2}+\sum_{i \geqslant 0} \mathcal{M}_{i}(T) \mathbb{R}^{2} .
$$

Now to establish the controllability of the linearized system, we distinguish between two cases.

CASE 1: $\gamma+\alpha \beta=0$. We begin with the "simplest" case when $\gamma+\alpha \beta=0$. We infer from (2.36) that $\bar{z}_{1}:=\bar{p}_{1}-\alpha \bar{w}_{1} \equiv 0$, hence $\bar{w}_{1}(T)=\alpha^{-1} \bar{p}_{1}(T)=0$ and $\ddot{\bar{w}}_{1}(T)=\alpha^{-1} \ddot{\bar{p}}_{1}(T)=0$. It follows that

$$
A(T)=\ddot{A}(T)=0, B(T)=\ddot{B}(T)=0 \text { and } D(T)=\ddot{D}(T)=0 .
$$

On the other hand, $\dot{\bar{w}}_{1}(T)=\alpha^{-1} \dot{\bar{p}}_{1}(T)=\alpha^{-1}(2 \pi / T)^{2} \lambda \neq 0$, hence

$$
\dot{A}(T)=\dot{\bar{w}}_{1}(T) \mathbf{A}, \quad \dot{B}(T)=\dot{\bar{w}}_{1}(T) \mathbf{B}, \dot{D}(T)=\dot{w}_{1}(T) \mathbf{D} .
$$

It follows that

$$
\begin{aligned}
\mathcal{R}_{f(0)=0} \supset & \mathcal{C} \mathbb{R}^{2}+\sum_{0 \leqslant i \leqslant 2} \mathcal{M}_{i}(T) \mathbb{R}^{2} \\
= & \left(\begin{array}{c}
0 \\
\mathcal{J}^{-1} C
\end{array}\right) \mathbb{R}^{2}+\left(\begin{array}{c}
\mathcal{J}^{-1} C \\
0
\end{array}\right) \mathbb{R}^{2} \\
& \quad+\left(\begin{array}{c}
0 \\
\mathcal{J}^{-1}\left(\mathbf{B}+\mathbf{A} \mathcal{J}^{-1} C\right)
\end{array}\right) \mathbb{R}^{2}+\left(\begin{array}{c}
\mathcal{J}^{-1}\left[\mathcal{J} \mathbf{D} \mathcal{J}^{-1} C+2\left(\mathbf{B}+\mathbf{A} \mathcal{J}^{-1} C\right)\right] \\
0
\end{array}\right) \mathbb{R}^{2} \\
= & \mathbb{R}^{6}
\end{aligned}
$$


thanks to (2.42)-(2.43). We have proved that (2.48) is controllable.

CASE 2. $\gamma+\alpha \beta \neq 0$. We claim that for $T>0$ arbitrary chosen and $\lambda_{0}$ small enough, we have for $0<\lambda<\lambda_{0}$,

$$
\operatorname{rank}\left(\mathcal{C}, \mathcal{M}_{0}(T), \mathcal{M}_{1}(T), \mathcal{M}_{2}(T)\right)=6 .
$$

First, $\left\|\bar{p}_{1}\right\|_{W^{2, \infty}(0, T)}=O(\lambda)$ still with $\bar{p}_{1}(T)=\ddot{\bar{p}}_{1}(T)=0$. From (2.46)-(2.47), we infer with Gronwall lemma (for $\lambda_{0}$ small enough) that $\bar{w}_{1}$ is well defined on $[0, T]$ and that $\left\|\bar{w}_{1}\right\|_{L^{\infty}(0, T)}=O(\lambda)$. This also yields (with (2.46)) $\left\|\bar{w}_{1}\right\|_{W^{2, \infty}(0, T)}=O(\lambda)$. Next, integrating in $(2.46)$ over $(0, T)$ yields $\bar{w}_{1}(T)=O\left(\lambda^{2}\right)$. Finally, derivating in (2.46) gives $\ddot{\bar{w}}_{1}(T)=O\left(\lambda^{2}\right)$. We conclude that

$$
(A(T), B(T), \ddot{A}(T), \ddot{B}(T))=O\left(\lambda^{2}\right), \quad D(T)=0,
$$

while

$$
(\dot{A}(T), \dot{B}(T), \dot{D}(T))=\alpha^{-1}(2 \pi / T)^{2} \lambda(\mathbf{A}, \mathbf{B}, \mathbf{D})+O\left(\lambda^{4}\right),
$$

for $\dot{\bar{p}}_{1}(T)=\alpha \dot{\bar{w}}_{1}(T)+O\left(\lambda^{4}\right)$. It follows that

$$
\operatorname{rank}\left(\mathcal{C}, \mathcal{M}_{0}(T), \mathcal{M}_{1}(T), \mathcal{M}_{2}(T)\right)
$$

$$
\begin{aligned}
& =\operatorname{rank}\left[\left(\begin{array}{c}
0 \\
\mathcal{J}^{-1} C
\end{array}\right),\left(\begin{array}{c}
\mathcal{J}^{-1} C \\
0
\end{array}\right),\right. \\
& \left.\quad\left(\begin{array}{c}
0 \\
\mathcal{J}^{-1}\left(\mathbf{B}+\mathbf{A} \mathcal{J}^{-1} C\right)
\end{array}\right),\left(\begin{array}{c}
\mathcal{J}^{-1}\left[\mathcal{J} \mathbf{D} \mathcal{J}^{-1} C+2\left(\mathbf{B}+\mathbf{A} \mathcal{J}^{-1} C\right)\right] \\
0
\end{array}\right)\right] \mathbb{R}^{2} \\
& =6,
\end{aligned}
$$

for $0<\lambda<\lambda_{0}$ with $\lambda_{0}$ small enough. This proves that (2.48) is controllable.

2.3.3. Step 3. Local controllability of the nonlinear system. Let us introduce the Hilbert space

$$
\mathcal{H}:=\mathbb{R}^{3} \times \mathbb{R}^{3} \times\left\{f \in H^{2}\left(0, T, \mathbb{R}^{2}\right) ; f(0)=0\right\}
$$

endowed with its natural Hilbertian norm

$$
\|(q, p, f)\|_{\mathcal{H}}^{2}=|q|^{2}+|p|^{2}+\|f\|_{H^{2}(0, T)}^{2} .
$$

We denote by $B_{\mathcal{H}}(0, \delta)$ the open ball in $\mathcal{H}$ with center 0 and radius $\delta$, i.e.

$$
B_{\mathcal{H}}(0, \delta)=\left\{(q, p, f) \in \mathcal{H} ;\|(q, p, f)\|_{\mathcal{H}}<\delta\right\} .
$$

Let us introduce the map

$$
\begin{aligned}
\Gamma: B_{\mathcal{H}}(0, \delta) & \rightarrow \mathbb{R}^{12} \\
\left(q_{0}, p_{0}, f\right) & \mapsto\left(q_{0}, p_{0}, q(T), p(T)\right)
\end{aligned}
$$

where $(q(t), p(t))$ denotes the solution of

$$
\begin{aligned}
\dot{q} & =\mathcal{Q}(q) p, \\
\mathcal{J} \dot{p} & =C(\dot{\bar{w}}+\dot{f})+F(p, \bar{w}+f), \\
(q(0), p(0)) & =\left(q_{0}, p_{0}\right) .
\end{aligned}
$$

Note that $\Gamma$ is well defined for $\delta>0$ small enough. Using the Sobolev embedding $H^{2}(0, T) \subset W^{1, \infty}(0, T)$ combined to [22, Theorem 1], we infer that $\Gamma$ is of class $C^{1}$ on $B_{\mathcal{H}}(0, \delta)$ and that its tangent linear map at the origin is given by

$$
d \Gamma(0)\left(z_{0}, k_{0}, f\right)=\left(z_{0}, k_{0}, z(T), k(T)\right),
$$


where $(z(t), k(t))$ solves the system

$$
\begin{aligned}
\left(\begin{array}{c}
\dot{z} \\
\dot{k}
\end{array}\right) & =\mathcal{A}(t)\left(\begin{array}{c}
z \\
k
\end{array}\right)+\mathcal{B}(t)\left(\begin{array}{l}
f_{1} \\
f_{2}
\end{array}\right)+\mathcal{C}\left(\begin{array}{l}
\dot{f}_{1} \\
\dot{f}_{2}
\end{array}\right), \\
\left(\begin{array}{c}
z(0) \\
k(0)
\end{array}\right) & =\left(\begin{array}{c}
z_{0} \\
k_{0}
\end{array}\right) .
\end{aligned}
$$

We know from Step 2 that (2.66)-(2.67) is controllable, so that $d \Gamma(0)$ is onto. Let $V:=$ (ker $d \Gamma(0))^{\perp}$ denote the orthogonal complement of ker $d \Gamma(0)$ in $\mathcal{H}$. Clearly $d \Gamma_{\mid V}(0)$ is invertible, and therefore it follows from the inverse function theorem that the map $\Gamma_{\mid V}: V \rightarrow \mathbb{R}^{12}$ is locally invertible at the origin. More precisely, there exists a number $\delta>0$ and an open set $\Omega \subset \mathbb{R}^{12}$ containing 0 such that the map $\Gamma: B_{\mathcal{H}}(0, \delta) \cap V \rightarrow \Omega$ is well-defined, of class $C^{1}$, invertible, and with an inverse map of class $C^{1}$. Let us denote this inverse map by $\Gamma^{-1}$, and let us write $\Gamma^{-1}\left(q_{0}, p_{0}, q_{T}, p_{T}\right)=\left(q_{0}, p_{0}, f\left(q_{0}, p_{0}, q_{T}, p_{T}\right)\right)$. Finally, let us set $w=\bar{w}+f$. (Note that $w(0)=0$.) Then, for $\eta>0$ small enough, we have that

$$
w \in C^{1}\left(B_{\mathbb{R}^{12}}(0, \eta), H^{2}\left(0, T, \mathbb{R}^{2}\right)\right)
$$

and that for $\left\|\left(q_{0}, p_{0}, q_{T}, p_{T}\right)\right\|<\eta$, the solution $(q(t), p(t))$ of the system

$$
\begin{aligned}
\dot{q} & =\mathcal{Q}(q) p, \\
\mathcal{J} \dot{p} & =C \dot{w}+F(p, w), \\
(q(0), p(0)) & =\left(q_{0}, p_{0}\right)
\end{aligned}
$$

satisfies $(q(T), p(T))=\left(q_{T}, p_{T}\right)$. The proof of Theorem 2.9 is complete.

\subsection{A global steady-state controllability result.}

Theorem 2.9 is a local controllability result. A global controllability result may be obtained when $\gamma+\alpha \beta=0$.

Theorem 2.14. If $\gamma+\alpha \beta=0$ and

$$
\begin{aligned}
& \operatorname{rank}\left(C, \frac{1}{2} \mathcal{J} \mathbf{D} \mathcal{J}^{-1} C+\mathbf{B}+\mathbf{A} \mathcal{J}^{-1} C\right)=3, \\
& \operatorname{rank}\left(\mathbf{B}+\mathbf{A} \mathcal{J}^{-1} C, \mathbf{A} \mathcal{J}^{-1}\left(\mathbf{B}+\mathbf{A} \mathcal{J}^{-1} C\right)\right)=3,
\end{aligned}
$$

then for any $\left(q_{0}, q_{T}\right) \in \mathbb{R}^{6}$ there exists a time $T>0$ and a control input $w \in H^{2}\left(0, T ; \mathbb{R}^{2}\right) \cap$ $H_{0}^{1}\left(0, T ; \mathbb{R}^{2}\right)$ driving the system

$$
\begin{aligned}
\dot{q} & =\mathcal{Q}(q) p, \\
\mathcal{J} \dot{p} & =C \dot{w}+F(p, w)
\end{aligned}
$$

from $\left(q_{0}, 0\right)$ at $t=0$ to $\left(q_{T}, 0\right)$ at $t=T$.

Proof. It may be assumed without loss of generality that $q_{0}=(0,0,0)$. We first establish a local controllability result around the equilibrium point $\left(q_{0}, 0\right)$. This is done along the same lines as for Theorem 2.9, using again the return method with the same reference trajectory $(\bar{q}, \bar{p}, \bar{w}) . \quad\left(\right.$ Note that $\bar{w}(0)=\bar{w}(T)=0$.) However, the new constraint $w \in H_{0}^{1}\left(0, T ; \mathbb{R}^{2}\right)$ impose to consider the reachable set $\mathcal{R}_{f(0)=f(T)=0}$ instead of the reachable set $\mathcal{R}_{f(0)=0}$. Recall that from Proposition 2.12

$$
\mathcal{R}_{f(0)=f(T)=0}=\sum_{i \geqslant 0} \mathcal{M}_{i}(T) \mathbb{R}^{2},
$$


where the sequence of matrices $\left(\mathcal{M}_{i}\right)_{i \geqslant 0}$ is the one associated with the pair $(\mathcal{A}, \mathcal{B}+\mathcal{A C})$. As a continuation of the computations performed in the proof of Theorem 2.9, we obtain

$$
\mathcal{M}_{3}(t)=\left(\begin{array}{c}
-\ddot{D} \mathcal{J}^{-1} C+\frac{d}{d t}\left\{D\left[D \mathcal{J}^{-1} C+\mathcal{J}^{-1}\left(B+A \mathcal{J}^{-1} C\right)\right]\right\} \\
-D\left\{-\dot{D} \mathcal{J}^{-1} C+D\left[D \mathcal{J}^{-1} C+\mathcal{J}^{-1}\left(B+A \mathcal{J}^{-1} C\right)\right]\right. \\
\left.-\mathcal{J}^{-1}\left[2\left(\dot{B}+\dot{A} \mathcal{J}^{-1} C\right)-A \mathcal{J}^{-1}\left(B+A \mathcal{J}^{-1} C\right)\right]\right\} \\
-\mathcal{J}^{-1}\left\{3\left[\ddot{B}+\ddot{A} \mathcal{J}^{-1} C\right]-2 \frac{d}{d t}\left[A \mathcal{J}^{-1}\left(B+A \mathcal{J}^{-1} C\right)\right]\right. \\
\left.-A \mathcal{J}^{-1}\left[\dot{B}+\dot{A} \mathcal{J}^{-1} C-A \mathcal{J}^{-1}\left(B+A \mathcal{J}^{-1} C\right)\right]\right\} \\
\mathcal{J}^{-1}\left\{\dddot{B}+\dddot{A} \mathcal{J}^{-1} C-\frac{d^{2}}{d t^{2}}\left[A \mathcal{J}^{-1}\left(B+A \mathcal{J}^{-1} C\right)\right]\right. \\
-\frac{d}{d t}\left(A \mathcal{J}^{-1}\left[\dot{B}+\dot{A} \mathcal{J}^{-1} C-A \mathcal{J}^{-1}\left(B+A \mathcal{J}^{-1} C\right)\right]\right) \\
-A \mathcal{J}^{-1}\left[\ddot{B}+\ddot{A} \mathcal{J}^{-1} C-\frac{d}{d t}\left[A \mathcal{J}^{-1}\left(B+A \mathcal{J}^{-1} C\right)\right]\right. \\
\left.\left.+\dot{B}+\dot{A} \mathcal{J}^{-1} C-A \mathcal{J}^{-1}\left(B+A \mathcal{J}^{-1} C\right)\right]\right\}
\end{array}\right)
$$

Using (2.61)-(2.62), we obtain

$$
\mathcal{M}_{3}(T)=\left(\begin{array}{l}
0 \\
\mathcal{J}^{-1}\left\{\dddot{B}(T)+\dddot{A}(T) \mathcal{J}^{-1} C-3 \dot{A}(T) \mathcal{J}^{-1}\left[\dot{B}(T)+\dot{A}(T) \mathcal{J}^{-1} C\right]\right\}
\end{array}\right) .
$$

Since $\dot{\bar{w}}_{1}(T)=\alpha^{-1} \dot{\bar{p}}_{1}(T)$ and $\frac{\bar{w}}{1}_{1}(T)=\alpha^{-1} \dddot{\bar{p}}_{1}(T)$, we infer that

$$
\begin{aligned}
\mathcal{R}_{f(0)=f(T)=0} \supset \sum_{0 \leqslant i \leqslant 3} \mathcal{M}_{i}(T) \mathbb{R}^{2} \\
=\left(\begin{array}{c}
\mathcal{J}^{-1} C \\
0
\end{array}\right) \mathbb{R}^{2}+\left(\begin{array}{c}
0 \\
\mathcal{J}^{-1}\left(\mathbf{B}+\mathbf{A} \mathcal{J}^{-1} C\right)
\end{array}\right) \mathbb{R}^{2} \\
\quad \quad+\left(\begin{array}{c}
\mathcal{J}^{-1}\left(\mathcal{J} \mathbf{D} \mathcal{J}^{-1} C+2\left(\mathbf{B}+\mathbf{A} \mathcal{J}^{-1} C\right)\right) \\
0
\end{array}\right) \mathbb{R}^{2} \\
\quad \quad+\left(\begin{array}{c}
0 \\
\mathcal{J}^{-1} \mathbf{A} \mathcal{J}^{-1}\left(\mathbf{B}+\mathbf{A} \mathcal{J}^{-1} C\right)
\end{array}\right) \mathbb{R}^{2} \\
\\
\quad=\mathbb{R}^{6},
\end{aligned}
$$

by (2.72)-(2.73). A local controllability may be deduced as in Theorem 2.9. Since $w$ vanishes at $t=0, T$, it follows from an iterated application of the previous result that any state of the form $(0,0, \theta, 0,0,0), \theta \in[0,2 \pi)$, may be reached from 0 . On the other hand, a (long) longitudinal displacement along the $y_{1}$-axis from $(q, p)=0$ to $(q, p)=$ $(L, 0,0,0,0,0)$ may be obtained by taking any $w=\left(w_{1}, 0\right) \in H^{2}\left(0, T, \mathbb{R}^{2}\right) \cap H_{0}^{1}\left(0, T, \mathbb{R}^{2}\right)$ with

$$
\int_{0}^{T} w_{1}(t) d t=\frac{L}{\alpha}
$$

A trajectory from the origin to any state $\left(q^{T}, 0\right)=\left(h_{1}^{T}, h_{2}^{T}, \theta^{T}, 0,0,0\right)$ may therefore be obtained as a concatenation of

(1) a trajectory from the origin to $(0,0, \theta, 0,0,0)$ with $\theta=\arg \left(h_{1}^{T}+i h_{2}^{T}\right)$;

(2) a straight line from $(0,0, \theta, 0,0,0)$ to $\left(h_{1}^{T}, h_{2}^{T}, \theta, 0,0,0\right)$;

(3) a trajectory from $\left(h_{1}^{T}, h_{2}^{T}, \theta, 0,0,0\right)$ to $\left(q^{T}, 0\right)$.

The proof of Theorem 2.14 is complete. 


\section{Wellposedness of THE SYSTEM (1.18)-(1.25) With VORTiCity}

In the previous section we considered the system (1.18)-(1.25) assuming that no vorticity was present. In this section, we prove that in presence of vorticity one can still construct a regular solution of (1.18)-(1.25) having a prescribed normal velocity, as for the solution we had constructed in Section 2. Using the results of Sections 2 and 3 and a time-scaling argument, we shall derive in Section 4 a controllability result for the whole system (with vorticity).

\subsection{Statements of the results.}

We are concerned with the existence of solutions to the following system:

$$
\begin{aligned}
\frac{\partial v}{\partial t}+\left[\left(v-l-r y^{\perp}\right) \cdot \nabla\right] v+r v^{\perp}+\nabla q=0 & \text { in }[0, T] \times \Omega, \\
\operatorname{div} v=0 & \text { in }[0, T] \times \Omega, \\
v \cdot n=\left(l+r y^{\perp}\right) \cdot n+\sum_{1 \leqslant j \leqslant m} w_{j}(t) \chi_{j}(y) & \text { on }[0, T] \times \partial \Omega, \\
\lim _{|y| \rightarrow \infty} v(t, y)=0 & \text { in }[0, T], \\
m i=\int_{\partial \Omega} q n d \sigma-m r l^{\perp} & \text { in }[0, T], \\
J \dot{r}=\int_{\partial \Omega} q n \cdot y^{\perp} d \sigma & \text { in }[0, T], \\
v(0, y)=v_{0}(y) & \text { in } \Omega, \\
(l(0), r(0))=\left(l_{0}, r_{0}\right) . &
\end{aligned}
$$

Once $(l, r)$ is known, the motion of the boat is described by the system

$$
\begin{aligned}
\dot{h} & =Q(\theta) l, \\
\dot{\theta} & =r, \\
(h(0), \theta(0)) & =\left(h_{0}, \theta_{0}\right) .
\end{aligned}
$$

For $k \in \mathbb{N}$ and $\alpha \in(0,1)$, let $C^{k, \alpha}(\bar{\Omega})$ denote the classical Hölder space endowed with the norm

$$
\|f\|_{C^{k, \alpha}(\bar{\Omega})}=\sum_{\beta \in \mathbb{N}^{2},|\beta| \leqslant k}\left(\left\|\partial^{\beta} f\right\|_{L^{\infty}(\Omega)}+\left|\partial^{\beta} f\right|_{0, \alpha}\right)
$$

where

$$
|f|_{0, \alpha}=\sup \left\{\frac{|f(x)-f(y)|}{|x-y|^{\alpha}} ; \quad x \in \bar{\Omega}, y \in \bar{\Omega}, x \neq y\right\},
$$

and for any $\theta>0$ and any $p \in[1, \infty)$, let $L_{\theta}^{p}(\Omega)$ denote the weighted space $L^{p}(\Omega ;(1+$ $\left.|y|^{\theta}\right) d y$ ) endowed with the norm

$$
\|f\|_{L_{\theta}^{p}(\Omega)}=\left(\int_{\Omega}|f(y)|^{p}\left(1+|y|^{\theta}\right) d y\right)^{\frac{1}{p}} .
$$

We prove in this section the following 
Proposition 3.1. Let $k \in \mathbb{N}, \alpha \in(0,1), \theta \in(2, \infty)$, and $T>0$. Assume given $\left(l_{0}, r_{0}\right) \in$ $\mathbb{R}^{3}, \omega_{0} \in C^{k, \alpha}(\bar{\Omega}) \cap L_{\theta}^{1}(\Omega)$, and a control function $w \in C^{1}\left([0, T], \mathbb{R}^{m}\right)$ (not necessarily such that $w(0)=0)$. Assume finally that the initial velocity field $v_{0} \in C^{k+1, \alpha}(\bar{\Omega})$ fulfills the following compatibility conditions

$$
\left\{\begin{array}{l}
\operatorname{curl} v_{0}=\omega_{0} \text { in } \Omega \\
\operatorname{div} v_{0}=0 \text { in } \Omega \\
v_{0} \cdot n=\left(l_{0}+r_{0} y^{\perp}\right) \cdot n+\sum_{j=1}^{m} w_{j}(0) \chi_{j} \quad \text { on } \partial \Omega \\
\lim _{|y| \rightarrow \infty} v_{0}(y)=0 \\
\int_{\partial \Omega} v_{0}(y) \cdot \tau d \sigma=-\int_{\Omega} \omega_{0}(y) d y
\end{array}\right.
$$

Then there exists a solution $(v, q, l, r)$ of (3.1)-(3.8) in the class

$$
\begin{aligned}
& v \in L^{\infty}\left(0, T ; C^{k+1, \alpha}(\bar{\Omega})\right) \cap H^{1}\left(0, T ; L^{2}(\Omega)\right), \quad \nabla v \in L^{\infty}\left(0, T ; L_{2}^{2}(\Omega)\right), \\
& \nabla q \in L^{\infty}\left(0, T ; L^{2}(\Omega)\right), \\
& (l, r) \in W^{1, \infty}\left(0, T ; \mathbb{R}^{3}\right) .
\end{aligned}
$$

Moreover, this solution satisfies for some constant $C>0$ (depending on the geometry, $\|w\|_{C^{1}},\left\|\omega_{0}\right\|_{C^{k, \alpha}(\bar{\Omega})},\left\|\omega_{0}\right\|_{L_{\theta}^{1}(\Omega)}$, and $\left|\left(l_{0}, r_{0}\right)\right|$ increasingly) that

$$
\|(l, r)-(\bar{l}, \bar{r})\|_{L^{\infty}(0, T)}+\|v(t)-\bar{v}(t)\|_{L^{\infty}\left(0, T ; C^{k+1, \alpha}(\bar{\Omega})\right)} \leqslant C\left(\left\|\omega_{0}\right\|_{C^{k, \alpha}(\bar{\Omega})}+\left\|\omega_{0}\right\|_{L_{\theta}^{1}(\Omega)}\right),
$$

where $(\bar{l}, \bar{r}, \bar{v})$ is the potential solution of (3.1)-(3.8) associated with $l_{0}, r_{0}, w$, and $\bar{\omega}_{0}=0$.

Remark 3.2. (1) The above solution $v$ is of course not unique, because we can fix to some extent the vorticity at points entering the fluid domain (see [11]). What we show is that for some reasonable choice of the vorticity on that part of $\partial \Omega$, we can have a regular solution of the nonhomogenous boundary value problem (3.1)-(3.8).

(2) We do not assume here that $w(0)=0$, as we shall consider later maximal solutions obtained by concatenation of solutions over time.

\subsection{Proof of Proposition 3.1.}

3.2.1. Notations. Let $\pi$ be a continuous linear extension operator from functions defined in $\Omega$ to functions defined in $\mathbb{R}^{2}$, which maps $C^{k, \alpha}(\bar{\Omega})$ to $C^{k, \alpha}\left(\mathbb{R}^{2}\right)$ for all $k \in \mathbb{N}$ and all $\alpha \in(0,1)$, and the space $\mathcal{L} \mathcal{L}(\Omega)$ of log-Lipschitz functions on $\Omega$ to $\mathcal{L} \mathcal{L}\left(\mathbb{R}^{2}\right)$. (The construction of such an "universal" extension operator is classical, see e.g. [23].)

We may also ask that $\pi$ preserves the divergence-free character (acting on the stream function if necessary).

We will use again the functions $\Phi_{i}, 1 \leqslant i \leqslant 3$ and $\Psi_{j}, 1 \leqslant j \leqslant m$ and the matrix $\mathcal{M}$ introduced in (2.11)-(2.13), (2.14)-(2.16), and (2.22), respectively.

3.2.2. Rephrasing the system. Now we rephrase a little bit the system. As before for potential flows, we show that the pressure solves some elliptic problem. Next, we replace the pressure by its expression in (3.5)-(3.6) to formulate in a new way the dynamics of the boat. The Laplacian of the pressure $q$ is given by

$$
\begin{aligned}
-\Delta q & =\operatorname{div}\left(\left[\left(v-l-r y^{\perp}\right) \cdot \nabla\right] v\right)+\operatorname{div}\left(r v^{\perp}\right) \\
& =\partial_{i}\left(v_{j} \partial_{j} v_{i}\right)-\partial_{i}\left(l_{j} \partial_{j} v_{i}\right)-\partial_{i}\left(r\left(y^{\perp}\right)_{j} \partial_{j} v_{i}\right)+r \operatorname{div}\left(v^{\perp}\right) \\
& \left.=\left(\partial_{i} v_{j}\right)\left(\partial_{j} v_{i}\right)-r\left(\partial_{i}\left(y^{\perp}\right)\right)_{j}\right) \partial_{j} v_{i}-r \omega \\
& =\left(\partial_{i} v_{j}\right)\left(\partial_{j} v_{i}\right)-r\left(\partial_{2} v_{1}-\partial_{1} v_{2}\right)-r \omega \\
& =\operatorname{tr}(\nabla v \cdot \nabla v),
\end{aligned}
$$


where we used Einstein's convention of repeated indices and the fact that $\operatorname{div}(v)=0$. Next, we compute the normal derivative of $q$ on $\partial \Omega$.

$$
\begin{aligned}
\frac{\partial q}{\partial n} & =-\left(\partial_{t} v+\left[\left(v-l-r y^{\perp}\right) \cdot \nabla\right] v+r v^{\perp}\right) \cdot n \\
& =-\partial_{t}\left(\left[l+r y^{\perp}+\sum_{j=1}^{m} w_{j} \nabla \Psi_{j}\right] \cdot n\right)-\left(\left[\left(v-l-r y^{\perp}\right) \cdot \nabla\right] v+r v^{\perp}\right) \cdot n \\
& =-\left(i+\dot{r} y^{\perp}\right) \cdot n-\sum_{j=1}^{m} \dot{w}_{j} \chi_{j}-\left(\left[\left(v-l-r y^{\perp}\right) \cdot \nabla\right] v+r v^{\perp}\right) \cdot n .
\end{aligned}
$$

Next, we introduce the function $\mu$ defined as the solution of the following elliptic problem:

$$
\begin{aligned}
-\Delta \mu=f:=\operatorname{tr}[\nabla v \cdot \nabla v], & \text { in }[0, T] \times \Omega \\
\frac{\partial \mu}{\partial n}=g:=-\sum_{j=1}^{m} \dot{w}_{j} \chi_{j}-\left(\left[\left(v-l-r y^{\perp}\right) \cdot \nabla\right] v+r v^{\perp}\right) \cdot n, & \text { on }[0, T] \times \partial \Omega .
\end{aligned}
$$

Note that for $v \in C^{k+1, \alpha}(\bar{\Omega})$ with $\nabla v \in L^{2}(\Omega) \cap L_{\theta^{\prime}}^{4}(\Omega)$ and $\theta^{\prime}>2$, then $f \in L^{1}(\Omega) \cap L_{\theta^{\prime}}^{2}(\Omega)$, $g \in C^{k, \alpha}(\partial \Omega)$ with the compatibility condition

$$
\int_{\Omega} f(y) d y=-\int_{\partial \Omega} g(y) d \sigma
$$

satisfied, and it follows from [1] that the problem (3.17)-(3.18) admits a solution $\mu \in$ $\widehat{H}^{1}(\Omega)$, which is unique up to a constant, with

$$
\|\nabla \mu\|_{L^{2}(\Omega)} \leqslant C\left[\sum_{j=1}^{m}\left|\dot{w}_{j}\right|+\|v\|_{C^{k+1, \alpha}(\bar{\Omega})}^{2}+\|\nabla v\|_{L_{\theta^{\prime}}^{4}(\Omega)}^{2}+|(l, r)|^{2}\right] .
$$

We see that

Noting that

$$
\nabla q=\nabla \mu-\left(\nabla \Phi_{j}\right)_{j=1,2,3}\left(\begin{array}{l}
i \\
\dot{r}
\end{array}\right)
$$

$$
\int_{\partial \Omega} q K_{i} d \sigma=\int_{\Omega} \nabla q \cdot \nabla \Phi_{i} d y
$$

we deduce the following form of (3.5)-(3.6)

$$
\left(\left[\begin{array}{cc}
m \mathrm{Id}_{2} & 0 \\
0 & J
\end{array}\right]+\mathcal{M}\right)\left(\begin{array}{l}
i \\
\dot{r}
\end{array}\right)=\left(\int_{\Omega} \nabla \mu(t, y) \cdot \nabla \Phi_{i}(y) d y\right)_{i=1,2,3}-\left(\begin{array}{c}
m r l^{\perp} \\
0
\end{array}\right) .
$$

After these preliminaries, we prove Proposition 3.1 in several steps. First, we prove the local-in-time existence of solutions (that is, up to some time $T^{\prime}$ which may be less than $T$ ), by means of Schauder's fixed point theorem. Next, we prove that such a solution can be extended up to time $T$ by using some $a$ priori estimates.

3.2.3. The operator. We first define an operator whose fixed points will give local-in-time solutions of (3.20).

Introduce

$$
M:=e \cdot\left\|\hat{\omega}_{0}\right\|_{C^{k, \alpha}\left(\mathbb{R}^{2}\right)}+\left(1+2^{2 \theta}\right)\left\|\hat{\omega}_{0}\right\|_{L_{\theta}^{1}\left(\mathbb{R}^{2}\right)} .
$$

Here $\hat{\omega}_{0}$ stands for the extension $\pi\left(\omega_{0}\right)$ of $\omega_{0}$ (see (3.29) below). 
Given $T^{\prime} \in(0, T], N>0$, and $\theta>2$, we introduce the set

$$
\begin{aligned}
& \mathcal{C}:=\left\{(l, r, \omega) \in C^{0}\left(\left[0, T^{\prime}\right] ; \mathbb{R}^{2}\right) \times C^{0}\left(\left[0, T^{\prime}\right] ; \mathbb{R}\right) \times L^{\infty}\left(0, T^{\prime} ; C^{k, \alpha}(\bar{\Omega}) \cap L_{\theta}^{1}(\Omega)\right) ;\right. \\
& \left.\left\|l-l_{0}\right\|_{L^{\infty}\left(0, T^{\prime}\right)}+\left\|r-r_{0}\right\|_{L^{\infty}\left(0, T^{\prime}\right)} \leqslant N,\|\omega\|_{L^{\infty}\left(0, T^{\prime} ; C^{k, \alpha}(\bar{\Omega})\right)}+\|\omega\|_{L^{\infty}\left(0, T^{\prime} ; L_{\theta}^{1}(\Omega)\right)} \leqslant M\right\} .
\end{aligned}
$$

Let us now define the operator $\mathcal{T}$ on $\mathcal{C}$ : to any $(l, r, \omega) \in \mathcal{C}$, we associate

$$
\mathcal{T}(l, r, \omega):=(\hat{l}, \hat{r}, \hat{\omega}),
$$

as follows. First, we introduce the "fluid velocity" $v$ as the solution to the system

$$
\begin{aligned}
\operatorname{curl} v=\omega & \text { in }[0, T] \times \Omega, \\
\operatorname{div} v=0 & \text { in }[0, T] \times \Omega, \\
v \cdot n=\left(l+r y^{\perp}\right) \cdot n+\sum_{j=1}^{m} w_{j}(t) \chi_{j}(y) & \text { on }[0, T] \times \partial \Omega, \\
\int_{\partial \Omega} v(t, y) \cdot \tau d \sigma=-\int_{\Omega} \omega(t, y) d y & \text { in }[0, T], \\
\lim _{|y| \rightarrow \infty} v(t, y)=0 & \text { in }[0, T] .
\end{aligned}
$$

Next, we extend the velocity field and the initial vorticity by letting

$$
\begin{aligned}
\hat{v}(t, \cdot) & :=\pi[v(t, \cdot)], \\
\hat{\omega}_{0} & :=\pi\left[\omega_{0}\right] .
\end{aligned}
$$

The flow $\hat{\Phi}$ associated with $\hat{v}-l-r y^{\perp}$ is defined as the solution to the Cauchy problem

$$
\left\{\begin{aligned}
\frac{\partial}{\partial t} \hat{\Phi}(t, s, y) & =\hat{v}(t, \hat{\Phi}(t, s, y))-l(t)-r(t) \hat{\Phi}^{\perp}(t, s, y), \\
\hat{\Phi}(s, s, y) & =y
\end{aligned}\right.
$$

where $(t, s, y)$ ranges over $\left[0, T^{\prime}\right]^{2} \times \mathbb{R}^{2}$. The vorticity part of $\mathcal{T}(l, r, \omega)$ is then given by

$$
\hat{\omega}(t, y):=\hat{\omega}_{0}(\hat{\Phi}(0, t, y)) .
$$

Note that $\hat{\omega}$ satisfies

$$
\hat{\omega}_{t}+\left(\hat{v}-l-r y^{\perp}\right) \cdot \nabla \hat{\omega}=0 .
$$

Finally, to define the pair $(\hat{l}, \hat{r})$, we introduce the function $\mu:[0, T] \times \Omega \rightarrow \mathbb{R}$ which solves

$$
\begin{cases}-\Delta \mu=\operatorname{tr}[\nabla v \cdot \nabla v] & \text { in }[0, T] \times \Omega, \\ \frac{\partial \mu}{\partial n}=-\sum_{j=1}^{m} \dot{w}_{j} \chi_{j}-\left(\left[\left(v-l-r y^{\perp}\right) \cdot \nabla\right] v+r v^{\perp}\right) \cdot n, & \text { on }[0, T] \times \partial \Omega .\end{cases}
$$

We define $\hat{l}$ and $\hat{r}$ as follows:

$$
\begin{aligned}
& {\left[\begin{array}{l}
\hat{l}(t) \\
\hat{r}(t)
\end{array}\right]=\left[\begin{array}{l}
l_{0} \\
r_{0}
\end{array}\right]} \\
& +\left(\left[\begin{array}{cc}
m \operatorname{Id}_{2} & 0 \\
0 & J
\end{array}\right]+\mathcal{M}\right)^{-1} \int_{0}^{t}\left[\left(\int_{\Omega} \nabla \mu(\tau, y) \cdot \nabla \Phi_{i}(y) d y\right)_{i=1,2,3}-\left(\begin{array}{c}
m r l^{\perp} \\
0
\end{array}\right)\right] d \tau,
\end{aligned}
$$

This completes the definition of $\mathcal{T}$. 
3.2.4. Fixed point argument and local-in-time existence. Our first step consists in proving the following result.

Lemma 3.3. Given any $N>0$, there exists some time $T^{\prime}>0$ such that $\mathcal{T}$ has a fixed point in $\mathcal{C}$.

Proof of Lemma 3.3: Let

$$
\bar{N}:=N+\left|l_{0}\right|+\left|r_{0}\right|
$$

In the sequel, the various positive constants $C_{i}$ will depend on the geometry, on $(m, J)$ and on the size of the controls $\left\|w_{i}\right\|_{C^{1}}$ only (hence, possibly also on $\pi$, but not on $l_{0}, r_{0}$, $\omega_{0}, N$, etc.).

1. Let $(l, r, \omega) \in \mathcal{C}$. Consider $\check{v}:=v-\sum_{j=1}^{m} w_{j} \nabla \Psi_{j}$, where $v$ is defined by (3.23)-(3.27). It satisfies

$$
\begin{cases}\operatorname{curl} \check{v}=\omega & \text { in }\left[0, T^{\prime}\right] \times \Omega, \\ \operatorname{div} \check{v}=0 & \text { in }\left[0, T^{\prime}\right] \times \Omega, \\ \check{v} \cdot n=\left(l+r y^{\perp}\right) \cdot n & \text { on }\left[0, T^{\prime}\right] \times \partial \Omega \\ \int_{\partial \Omega} \check{v}(t, y) \cdot \tau d \sigma=-\int_{\Omega} \omega(t, y) d y & \text { in }\left[0, T^{\prime}\right] \\ \lim _{|y| \rightarrow \infty} \check{v}(t, y)=0 & \text { in }\left[0, T^{\prime}\right] .\end{cases}
$$

It follows from [19, Proposition 2.2] that for all $p \in(2, \infty)$,

$$
\|\breve{v}(t)\|_{W^{1, p}(\Omega)} \leqslant C\left(\|\omega(t)\|_{L^{1}(\Omega)}+\|\omega(t)\|_{L^{\infty}(\Omega)}+|(l(t), r(t))|\right) .
$$

Standard Schauder estimates give

$$
\begin{aligned}
\|\check{v}\|_{L^{\infty}\left(0, T^{\prime} ; C^{k+1, \alpha}(\bar{\Omega})\right) \quad \leqslant C} & \left(\|\omega\|_{L^{\infty}\left(0, T^{\prime} ; C^{k, \alpha}(\bar{\Omega})\right)}+\|l\|_{L^{\infty}\left(0, T^{\prime}\right)}+\|r\|_{L^{\infty}\left(0, T^{\prime}\right)}\right. \\
& \left.+\|\omega\|_{L^{\infty}\left(0, T^{\prime} ; L^{1}(\Omega)\right)}+\|\check{v}\|_{L^{\infty}\left(0, T^{\prime} ; C^{0, \alpha}(\bar{\Omega})\right)}\right) .
\end{aligned}
$$

Combining (3.34) with (3.35) and Sobolev embedding, we infer that

$$
\left\|v-\sum_{j=1}^{m} w_{j} \nabla \Psi_{j}\right\|_{L^{\infty}\left(0, T^{\prime} ; C^{k+1, \alpha}(\bar{\Omega})\right)} \leqslant C_{1}(\bar{N}+M),
$$

and consequently, using the continuity of $\pi$ we obtain

$$
\left\|\hat{v}-\sum_{j=1}^{m} w_{j} \pi\left(\nabla \Psi_{j}\right)\right\|_{L^{\infty}\left(0, T^{\prime} ; C^{k+1, \alpha}\left(\mathbb{R}^{2}\right)\right)} \leqslant\|\pi\| C_{1}(\bar{N}+M),
$$

where $\|\pi\|$ denotes the norm of $\pi$ as an operator in $\mathcal{L}\left(C^{k+1, \alpha}(\bar{\Omega}), C^{k+1, \alpha}\left(\mathbb{R}^{2}\right)\right)$. Therefore

$$
\|\hat{v}\|_{L^{\infty}\left(0, T^{\prime} ; C^{k+1, \alpha}\left(\mathbb{R}^{2}\right)\right)} \leqslant C_{2}(\bar{N}+M+1) .
$$

2. It follows from Gronwall's lemma (see also [3, Lemma 4.1.1]) that

$$
\|\hat{\omega}\|_{L^{\infty}\left(0, T^{\prime}, C^{k, \alpha}\left(\mathbb{R}^{2}\right)\right)} \leqslant\left\|\hat{\omega}_{0}\right\|_{C^{k, \alpha}\left(\mathbb{R}^{2}\right)} \exp \left(C_{3} T^{\prime}\left\|\nabla\left(\hat{v}-l-r y^{\perp}\right)\right\|_{L^{\infty}\left(0, T^{\prime}, C^{k-1, \alpha}\left(\mathbb{R}^{2}\right)\right.}\right),
$$

hence

$$
\|\hat{\omega}\|_{L^{\infty}\left(0, T^{\prime}, C^{k, \alpha}\left(\mathbb{R}^{2}\right)\right)} \leqslant\left\|\hat{\omega}_{0}\right\|_{C^{k, \alpha}\left(\mathbb{R}^{2}\right)} \exp \left(C_{4} T^{\prime}(\bar{N}+M+1)\right)
$$

Using (3.37) once again, we infer that

$$
\forall(s, t) \in\left[0, T^{\prime}\right]^{2}, \forall y \in \mathbb{R}^{2}, \quad|\hat{\Phi}(t, s, y)| \leqslant 2\left(|y|+C_{5} T^{\prime}(\bar{N}+M+1)\right) .
$$


It follows that for any $t \in\left[0, T^{\prime}\right]$, with Liouville's theorem,

$$
\begin{aligned}
\int_{\mathbb{R}^{2}}|\hat{\omega}(t, x)|\left(1+|x|^{\theta}\right) d x & =\int_{\mathbb{R}^{2}}\left|\hat{\omega}_{0}(y)\right|\left(1+|\hat{\Phi}(t, 0, y)|^{\theta}\right) d y \\
& \leqslant \int_{\mathbb{R}^{2}}\left|\hat{\omega}_{0}(y)\right|\left(1+2^{\theta}\left[|y|+C_{5} T^{\prime}(\bar{N}+M+1)\right]^{\theta}\right) d y \\
& \leqslant \int_{\mathbb{R}^{2}}\left|\hat{\omega}_{0}(y)\right|\left(1+2^{2 \theta-1}\left(1+\left[C_{5} T^{\prime}(\bar{N}+M+1)\right]^{\theta}\right)\left(1+|y|^{\theta}\right)\right) d y \\
& \leqslant\left(1+2^{2 \theta-1}\left(1+\left[C_{5} T^{\prime}(\bar{N}+M+1)\right]^{\theta}\right)\right)\left\|\hat{\omega}_{0}\right\|_{L_{\theta}^{1}} .
\end{aligned}
$$

Assuming that $C_{5} \geqslant C_{4}$, we infer that the condition about $\hat{\omega}$ for $\mathcal{T}(l, r, \omega)$ to belong to $\mathcal{C}$ is satisfied provided that

$$
T^{\prime} \leqslant \frac{1}{C_{5}(\bar{N}+M+1)}
$$

3. Let us turn our attention to $(\hat{l}, \hat{r})$. From $(3.26)$ and $[19$, Proofs of Proposition 2.2 and Lemma 2.6] we see that for a.e. $t \in\left(0, T^{\prime}\right), \check{v}(t) \in L^{2}(\Omega)$ and $(1+|y|) \nabla \check{v}(t) \in L^{p}(\Omega)$ for all $p \in[2, \theta]$ with

$$
\|\check{v}(t)\|_{L^{2}(\Omega)}+\|(1+|y|) \nabla \check{v}(t)\|_{L^{p}(\Omega)} \leqslant C_{6}\left(\|\omega(t)\|_{L^{\infty}(\Omega)}+\|\omega(t)\|_{L_{\theta}^{1}(\Omega)}+|(l(t), r(t))|\right) .
$$

An application of Hölder estimate with (3.34) and (3.42) gives that for some $\theta^{\prime} \in(2, \min (\theta, 7))$ and a.e. $t \in(0, T)$

$$
\|\nabla \check{v}(t)\|_{L_{\theta^{\prime}}^{4}(\Omega)} \leqslant C_{7}\left(\|\omega(t)\|_{L^{\infty}(\Omega)}+\|\omega(t)\|_{L_{\theta}^{1}(\Omega)}+|(l(t), r(t))|\right) .
$$

Combined to (2.19), this yields

$$
\|\nabla v\|_{L^{\infty}\left(0, T^{\prime}, L_{\theta^{\prime}}^{4}(\Omega)\right)} \leqslant C_{8}(\bar{N}+M+1)
$$

and then, with (3.37) and (3.19)

$$
\|\nabla \mu\|_{L^{\infty}\left(0, T^{\prime}, L^{2}(\Omega)\right)} \leqslant C_{9}(\bar{N}+M+1)^{2} .
$$

One deduces that

$$
\left\|(\hat{l}, \hat{r})-\left(l_{0}, r_{0}\right)\right\|_{\infty} \leqslant C_{10} T^{\prime}(\bar{N}+M+1)^{2} .
$$

Therefore, the condition about $(\hat{l}, \hat{r})$ for $\mathcal{T}(l, r, \omega)$ to belong to $\mathcal{C}$ is satisfied provided that

$$
T^{\prime} \leqslant \frac{N}{C_{10}(\bar{N}+M+1)^{2}} .
$$

Hence for $T^{\prime}$ satisfying (3.41) and (3.45), one has $\mathcal{T}(\mathcal{C}) \subset \mathcal{C}$.

4. It is easy to check that $\mathcal{C}$ is convex and closed for the uniform topology on $(l, r, \omega)$, i.e. in $E=L^{\infty}\left(0, T^{\prime}, \mathbb{R}^{2} \times \mathbb{R} \times L^{\infty}(\Omega)\right)$. We claim that $\mathcal{T}(\mathcal{C})$ is relatively compact in $E$. For the $(l, r)$ component, it is sufficient to use (3.33) and the compactness of the embedding $W^{1, \infty}\left(0, T^{\prime}\right) \subset C\left(\left[0, T^{\prime}\right]\right)$. For the $\omega$ component, this is established thanks to Aubin-Lions' Lemma (see e.g. [21]), (3.31), and the compactness of the embedding

$$
C^{0, \alpha}(\bar{\Omega}) \cap L_{\theta}^{1}(\Omega) \subset C^{0, \alpha^{\prime}}(\bar{\Omega}) \cap L_{\theta^{\prime}}^{1}(\Omega),
$$

for $0<\alpha^{\prime}<\alpha$, and $2<\theta^{\prime}<\theta$. 
Finally, let us show that $\mathcal{T}$ is continuous for the $L^{\infty}$ topology. Suppose that $\left(l_{n}, r_{n}, \omega_{n}\right)$ converges to $(l, r, \omega)$ uniformly. We notice that the set

$$
\mathcal{K}:=\left\{\omega \in C^{k, \alpha}(\bar{\Omega}) \cap L_{\theta}^{1}(\Omega),\|\omega\|_{C^{k, \alpha}(\bar{\Omega})}+\|\omega\|_{L_{\theta}^{1}(\Omega)} \leqslant M\right\},
$$

is compact for both the $L^{\infty}(\Omega)$ topology and the $C^{0, \alpha^{\prime}}(\bar{\Omega}) \cap L_{\theta^{\prime}}^{1}(\Omega)$ topology (where again $0<\alpha^{\prime}<\alpha$, and $2<\theta^{\prime}<\theta$ ). Consequently the map $\mathcal{K} \rightarrow \mathcal{K}, f \mapsto f$ is uniformly continuous in both directions. As a consequence, the uniform convergence of $\omega_{n}$ towards $\omega$ implies the convergence in $L^{\infty}\left(0, T^{\prime} ; C^{0, \alpha^{\prime}}(\bar{\Omega}) \cap L_{\theta^{\prime}}^{1}(\Omega)\right)$. Combined to the uniform convergence of $\left(l_{n}, r_{n}\right)$ to $(l, r)$, this yields

$$
\begin{aligned}
v_{n} & \rightarrow v \quad \text { in } L^{\infty}\left(0, T^{\prime} ; C^{1, \alpha^{\prime}}(\bar{\Omega})\right) \quad \forall \alpha^{\prime} \in(0, \alpha), \\
\text { and } \quad \nabla v_{n} & \rightarrow \nabla v \quad \text { in } L^{\infty}\left(0, T^{\prime} ; L_{\theta^{\prime}}^{4}(\Omega)\right) \quad \text { for some } \theta^{\prime} \in(2, \theta) .
\end{aligned}
$$

It follows that

$$
\begin{aligned}
\nabla \mu_{n} & \rightarrow \nabla \mu \quad \text { in } L^{\infty}\left(0, T^{\prime} ; L^{2}(\Omega)\right), \\
\text { hence } \quad\left(\hat{l}_{n}, \hat{r}_{n}\right) & \rightarrow(\hat{l}, \hat{r}) \quad \text { in } W^{1, \infty}\left(0, T^{\prime}\right) .
\end{aligned}
$$

On the other hand, $\hat{\Phi}_{n}(s, t, y) \rightarrow \hat{\Phi}(s, t, y)$ uniformly on compact sets of $\left[0, T^{\prime}\right]^{2} \times \mathbb{R}^{2}$, so that $\hat{\omega}_{n}(t, y) \rightarrow \hat{\omega}(t, y)$ uniformly on compact sets of $\left[0, T^{\prime}\right] \times \mathbb{R}^{2}$. Since $\mathcal{T}(\mathcal{C})$ is relatively compact in $E$, we conclude that $\hat{\omega}_{n} \rightarrow \hat{\omega}$ in $L^{\infty}\left(0, T^{\prime}, L^{\infty}(\Omega)\right)$. The continuity of $\mathcal{T}$ is proved. The conclusion of Lemma 3.3 follows then from Schauder's fixed point theorem.

Let us now check that the fixed point solution $(l, r, \omega)$ given in Lemma 3.3 yields a solution of (3.1)-(3.8). Let $v$ and $\mu$ be given by (3.23)-(3.27) and (3.32), respectively, and let

$$
q:=\mu-\left(\dot{l}_{1} \Phi_{1}+\dot{l}_{2} \Phi_{2}+\dot{r} \Phi_{3}\right) .
$$

Then (3.5)-(3.6) follows at once from (3.33). Using (3.25), (3.32) and (3.46) we see that for a.e. $t \in\left(0, T^{\prime}\right), q(t,$.$) satisfies$

$$
\begin{aligned}
-\Delta q & =\operatorname{div}\left(\left(v-l-r y^{\perp}\right) \cdot \nabla v+r v^{\perp}\right), \quad \text { in } \Omega \\
\frac{\partial q}{\partial n} & =-\left(v_{t}+\left(v-l-r y^{\perp}\right) \cdot \nabla v+r v^{\perp}\right) \cdot n, \quad \text { on } \partial \Omega .
\end{aligned}
$$

Introduce the function

$$
F(t, y):=v(t, y)-v_{0}(y)+\int_{0}^{t}\left[\left(v-l-r y^{\perp}\right) \cdot \nabla v+r v^{\perp}+\nabla q\right](s, y) d s .
$$

Then it follows from (3.31), (3.47), and (3.48) that

$$
\begin{aligned}
& \operatorname{div} F=0 \quad \text { in } \Omega \text {, } \\
& \operatorname{curl} F=0 \quad \text { in } \Omega \text {, } \\
& F \cdot n=0 \quad \text { on } \partial \Omega \text {. }
\end{aligned}
$$

Clearly $F(t,.) \in L^{2}(\Omega) \cap C_{l o c}^{k, \alpha}(\bar{\Omega})$ for a.e. $t \in\left(0, T^{\prime}\right)$. Pick such a time $t$. Using (3.49)(3.51), we see that $F(t,.) \in H^{1}(\Omega \cap B(0, R))$ for all $R>0$. It follows then from [19, Lemma 2.5] that

$$
\int_{\partial \Omega} F(t, y) \cdot \tau d \sigma=0
$$

Clearly, there exists some harmonic function $\psi$ on $\Omega$ such that $F(t, y)=\nabla \psi(y)$. From the proof of Lemma 2.2 we easily see that $\nabla \psi(y)$ has a limit as $|y| \rightarrow \infty$. That limit has to be 0 , for $F(t,.) \in L^{2}(\Omega)$. This shows that $F(t,)=$.0 for a.e. $t \in\left(0, T^{\prime}\right)$, hence $(v, q, l, r)$ 
satisfies (3.1)-(3.7). (3.8) follows from (3.33). This gives the local-in-time existence part of Proposition 3.1.

Remark 3.4. (1) If $k \geqslant 1$, it is possible to show that the operator $\mathcal{T}$ contracts for small $T$ in the norm $\|\cdot\|_{L^{\infty}\left(0, T ; C^{k-1, \alpha}(\bar{\Omega})\right)}$, using a priori estimates on $v$ in $C^{k+1, \alpha}(\bar{\Omega})$ and Gronwall's lemma.

(2) One easily sees that for all $\alpha^{\prime} \in(0, \alpha)$ and all $\theta^{\prime} \in(2, \theta)$ it holds

$$
\omega \in C\left(\left[0, T^{\prime}\right], C^{k, \alpha^{\prime}}(\bar{\Omega}) \cap L_{\theta^{\prime}}^{1}(\Omega)\right) \quad \text { and } \quad v \in C\left(\left[0, T^{\prime}\right], C^{k+1, \alpha^{\prime}}(\bar{\Omega})\right) .
$$

3.2.5. A priori estimates and global existence. Let us consider a solution of (3.1)-(3.8) defined on a maximal interval of existence $\left(0, T^{*}\right)$ (with $0<T^{*} \leqslant T$ ), fulfilling (3.13)(3.15) for all $T^{\prime}<T^{*}$, and satisfying as the solution constructed above that

$$
\operatorname{curl}(v)=\hat{\omega}_{\mid \Omega},
$$

where $\hat{\omega}(t, y)=\hat{\omega}_{0}(\hat{\Phi}(0, t, y))$ and $\hat{\Phi}$ denotes as above the flow associated with $\hat{\pi}(v)-l-r y^{\perp}$. In particular, $v$ satisfies

$$
\|\operatorname{curl}(v)\|_{L^{\infty}\left(0, T^{*}, L^{p}(\Omega)\right)} \leqslant C, \quad \forall p \in[1,+\infty] .
$$

We will establish an a priori estimate on $(l, r, \omega)$ in a suitable space. With Lemma 3.3 and a standard procedure, this will give that $T^{*}=T$.

1. First, we perform an energy estimate. Since the boundary condition is not homogeneous, the energy in $\Omega$ may not be conserved. From (3.1) we obviously obtain that

$\frac{\partial}{\partial t}\left(v-\sum_{j=1}^{m} w_{j} \nabla \Psi_{j}\right)+\left[\left(v-l-r y^{\perp}\right) \cdot \nabla\right] v+r v^{\perp}+\sum_{j=1}^{m} \dot{w}_{j} \nabla \Psi_{j}+\nabla q=0 \quad$ in $[0, T] \times \Omega$.

Multiplying by $\check{v}:=v-\sum_{j=1}^{m} w_{j} \nabla \Psi_{j}$ and integrating over $\Omega$, we obtain

$$
\begin{aligned}
& \frac{1}{2} \frac{d}{d t} \int_{\Omega} \check{v}(t, y)^{2} d y-\int_{\Omega} \frac{|v|^{2}}{2}(t, y) \operatorname{div}\left(v-l-r y^{\perp}\right) d y+\int_{\partial \Omega} \frac{|v|^{2}}{2}(t, y)\left(v-l-r y^{\perp}\right) \cdot n d \sigma \\
& -\sum_{j=1}^{m} w_{j}(t)\left\{-\int_{\Omega} v \cdot \nabla \Psi_{j} \operatorname{div}\left(v-l-r y^{\perp}\right) d y-\int_{\Omega}\left\{\left[\left(v-l-r y^{\perp}\right) \cdot \nabla\right] \nabla \Psi_{j}\right\} \cdot v d y\right. \\
& \left.\quad+\int_{\partial \Omega}\left[v \cdot \nabla \Psi_{j}\right]\left(v-l-r y^{\perp}\right) \cdot n d \sigma\right\} \\
& \quad-\sum_{j=1}^{m} w_{j}(t) \int_{\Omega} r v^{\perp} \cdot \nabla \Psi_{j} d y+\sum_{j=1}^{m} \int_{\Omega} \dot{w}_{j} \nabla \Psi_{j} \cdot \check{v}(t, y) d y+\int_{\Omega} \nabla q(t, y) \cdot \check{v}(t, y) d y \\
& =: I_{1}+I_{2}+\cdots+I_{7}=0 .
\end{aligned}
$$

The above computations are legitimate thanks to (3.13)-(3.15) (with $T$ replaced by any $\left.T^{\prime}<T^{*}\right)$. Note that $(3.26)$ is essential here to have a solution with a finite energy.

a. From $\operatorname{div}\left(v-l-r y^{\perp}\right)=0$ we infer that $I_{2}=0$ and that the first integral term in $I_{4}$ is nul. 
b. We integrate by parts in $I_{7}$ to get with (3.5)-(3.6)

$$
\begin{aligned}
I_{7} & =\int_{\partial \Omega} q(t, y) \check{v}(t, y) \cdot n d \sigma \\
& =\int_{\partial \Omega} q(t, y) l(t) \cdot n d \sigma+\int_{\partial \Omega} q(t, y) r(t) y^{\perp} \cdot n d \sigma \\
& =\frac{1}{2} \frac{d}{d t}\left(m l^{2}+J r^{2}\right) .
\end{aligned}
$$

c. Integrating by parts in $I_{6}$ yields

$$
I_{6}=\sum_{j=1}^{m} \int_{\partial \Omega} \dot{w}_{j} \Psi_{j}(y) \check{v}(t, y) \cdot n d \sigma=\sum_{j=1}^{m} \int_{\partial \Omega} \dot{w}_{j} \Psi_{j}(y)\left(l+r y^{\perp}\right) \cdot n d \sigma .
$$

d. Denote by $E$ the total energy of the system, namely

$$
E:=\frac{1}{2}\left(\int_{\Omega}|\check{v}|^{2}(t, y) d y+m l^{2}+J r^{2}\right)
$$

Since

$$
\|v\|_{L^{2}}^{2} \leqslant(m+1)\left(\|\check{v}\|_{L^{2}}^{2}+\sum_{j=1}^{m} w_{j}^{2}\left\|\nabla \Psi_{j}\right\|_{L^{2}}^{2}\right),
$$

the total energy allows to control $v$ in $L^{2}(\Omega)$ as well. Consequently, we have

$$
\frac{d}{d t} E=\int_{\partial \Omega} v(t, y) \cdot\left[-\frac{1}{2} v(t, y)+\sum_{j=1}^{m} w_{j}(t) \nabla \Psi_{j}(y)\right]\left(\sum_{i=1}^{m} w_{i}(t) \chi_{i}(y)\right) d \sigma+F,
$$

where $F$ satisfies

$$
F \leqslant C(1+E)
$$

for some constant $C>0$ depending on $\left\|\Psi_{j}\right\|_{L^{2}(\partial \Omega)},\left\|\nabla \Psi_{j}\right\|_{L^{2}(\Omega)},\left\|y \nabla^{2} \Psi_{j}\right\|_{L^{2}(\Omega)},\left\|\nabla^{2} \Psi_{j}\right\|_{L^{\infty}(\Omega)}$ (which are finite thanks to $(2.19)$ ) and $\left\|w_{j}\right\|_{C^{1}([0, T])}$ for $1 \leqslant j \leqslant m$.

2. We infer from (3.34) that

$$
\|v\|_{C(\bar{\Omega})} \leqslant C\left(\|\omega\|_{L^{1}(\Omega)}+\|\omega\|_{L^{\infty}(\Omega)}+|(l, r)|+\|w\|_{C^{1}([0, T])}\right) .
$$

It follows that

$$
\frac{d}{d t} E \leqslant C(1+E) .
$$

Hence, by Gronwall's lemma, $E$ remains bounded up to time $T^{*}$.

3. In particular $(l, r)$ is bounded up to time $T^{*}$, hence $v \in L^{\infty}\left(0, T^{*} ; L^{\infty}(\Omega)\right)$. We infer from [13, Lemma 2.4] that for some constant $K>0$

$$
\|v(t)\|_{\mathcal{L L}} \leqslant K \quad \text { for a.e. } t \in\left(0, T^{*}\right) .
$$

An application of the well-known Wolibner-Yudovich theory yields that the flow $\Phi$ of $\pi(v)-l-r y^{\perp}$ is defined up to time $T^{*}>0$ and that, as a function of $(t, s, y)$, it is Hölder continuous with Hölder index $\delta=\exp \left(-T^{*} K\right)$ (see [13, Lemma 2.5]). It follows that $\omega \in L^{\infty}\left(0, T^{*} ; C^{0, \alpha \delta}(\bar{\Omega})\right)$, and therefore $v \in L^{\infty}\left(0, T^{*} ; C^{1, \alpha \delta}(\bar{\Omega})\right)$. Consequently, $\omega \in$ $L^{\infty}\left(0, T^{*} ; C^{0, \alpha}(\bar{\Omega})\right)$. Using a straightforward bootstrap argument, we conclude that $v \in$ $L^{\infty}\left(0, T^{*} ; C^{k+1, \alpha}(\bar{\Omega})\right)$. This yields $\omega \in L^{\infty}\left(0, T^{*} ; C^{k, \alpha}(\bar{\Omega}) \cap L_{\theta}^{1}(\Omega)\right)$. If $T^{*}<T$, we may construct a solution defined on an interval $\left(0, T^{*}+\varepsilon\right)$ (for some $\varepsilon>0$ ), which contradicts the fact that $T^{*}$ was maximal. We conclude that $T^{*}=T$. 
3.2.6. Proof of (3.16). First, we notice that $(\bar{l}, \bar{r}, \bar{v})$ is obtained in the following way. Since $\bar{\omega}_{0}=0, \pi\left(\bar{\omega}_{0}\right)=0$ on $\mathbb{R}^{2}$, hence with $(3.30)$ the vorticity $\bar{\omega}$ is null as well as the circulation $\int_{\partial \Omega} \bar{v} \cdot \tau d \sigma$. Thus (3.23)-(3.27) gives

$$
\bar{v}(t, y)=\left(\nabla \Phi_{j}(y)\right)_{j=1,2,3}\left(\begin{array}{c}
\bar{l}(t) \\
\bar{r}(t)
\end{array}\right)+\sum_{j=1}^{m} w_{j}(t) \nabla \Psi_{j}(y) .
$$

It follows from Proposition 2.4 that $(\bar{l}, \bar{r})$ satisfies the (algebraic) ODE (5.32)-(5.34), whose solution is unique.

Now consider a solution $(l, r, \omega)$ as constructed above. Following Section 3.2.5, we see that for some constant $C=C\left(\left\|\omega_{0}\right\|_{C^{k, \alpha}(\bar{\Omega})},\left\|\omega_{0}\right\|_{L_{\theta}^{1}(\Omega)},\left|l_{0}\right|,\left|r_{0}\right|\right)$ (also depending on the geometry and $w_{i}$ ), growing in its arguments, we have

$$
\|\omega\|_{L^{\infty}\left(0, T, C^{k, \alpha}(\bar{\Omega})\right)}+\|\omega\|_{L^{\infty}\left(0, T, L_{\theta}^{1}(\Omega)\right)} \leqslant C \mathcal{N}
$$

where

$$
\mathcal{N}:=\left\|\omega_{0}\right\|_{C^{k, \alpha}(\bar{\Omega})}+\left\|\omega_{0}\right\|_{L_{\theta}^{1}(\Omega)} .
$$

Now, from (3.23)-(3.27), we easily infer that

$$
\|v(t)-\bar{v}(t)\|_{C^{k+1, \alpha}(\bar{\Omega})}+\|\nabla v(t)-\nabla \bar{v}(t)\|_{L_{\theta^{\prime}}^{4}(\Omega)} \leqslant C(\mathcal{N}+|(l(t), r(t))-(\bar{l}(t), \bar{r}(t))|) .
$$

Injecting in (3.32), we deduce that

$$
\|\nabla \mu(t)-\nabla \bar{\mu}(t)\|_{L^{2}(\Omega)} \leqslant C(\mathcal{N}+|(l(t), r(t))-(\bar{l}(t), \bar{r}(t))|) .
$$

Combined with (3.20), this gives

$$
|(\dot{l}(t), \dot{r}(t))-(\dot{\bar{l}}(t), \dot{\bar{r}}(t))| \leqslant C(\mathcal{N}+|(l(t), r(t))-(\bar{l}(t), \bar{r}(t))|) .
$$

Hence the claim for $|(l, r)-(\bar{l}, \bar{r})|$ follows from Gronwall's lemma. The proof of Proposition 3.1 is complete.

The next result is concerned with the uniqueness of the solution $(v, q, l, r)$ of $(3.1)-(3.8)$, when the vorticity $\omega=\operatorname{curl} v$ fulfills

$$
\omega(t, y):=\pi\left(\omega_{0}\right)(\Phi(0, t, y))
$$

with the flow $\Phi$ defined by

$$
\left\{\begin{aligned}
\frac{\partial}{\partial t} \Phi(t, s, y) & =\pi(v)(t, \Phi(t, s, y))-l(t)-r(t) \Phi^{\perp}(t, s, y), \\
\Phi(s, s, y) & =y .
\end{aligned}\right.
$$

Proposition 3.5. Let $l_{0}, r_{0}, \omega_{0}, v_{0}$ be as in Proposition 3.1 and assume further that $\omega_{0} \in$ $W^{1, \infty}(\Omega)$. Then the solution $(v, q, l, r, \omega)$ of (3.1)-(3.8) and (3.53)-(3.54) is unique in the class (3.13)-(3.15). On the other hand, for any given initial data $\left(l_{0}, r_{0}, \omega_{0}\right)$ as above, the map $w \in H^{2}(0, T) \mapsto(l, r) \in C([0, T])$ is continuous.

Proof: Assume given two solutions $\left(v^{1}, q^{1}, l^{1}, r^{1}\right)$ and $\left(v^{2}, q^{2}, l^{2}, r^{2}\right)$ of (3.1)-(3.8), corresponding to the same initial data $\left(l_{0}, r_{0}, v_{0}\right)$ and to the same control $w$, in the class (3.13)-(3.15), with $\omega^{i}=\operatorname{curl} v^{i}$ fulfilling for $i=1,2$

$$
\omega^{i}(t, y):=\pi\left(\omega_{0}\right)\left(\Phi^{i}(0, t, y)\right)
$$

where $\Phi^{i}$ denotes the solution to

$$
\left\{\begin{aligned}
\frac{\partial}{\partial t} \Phi^{i}(t, s, y) & =\pi\left(v^{i}\right)\left(t, \Phi^{i}(t, s, y)\right)-l^{i}(t)-r^{i}(t)\left(\Phi^{i}\right)^{\perp}(t, s, y), \\
\Phi^{i}(s, s, y) & =y .
\end{aligned}\right.
$$


We introduce the functions

$$
v=v^{1}-v^{2}, \quad q=q^{1}-q^{2}, \quad r=r^{1}-r^{2}, \quad l=l^{1}-l^{2},
$$

which satisfy the following system

$$
\begin{aligned}
\frac{\partial v}{\partial t}+\left(\left(v^{1}-l^{1}-r^{1} y^{\perp}\right) \cdot \nabla\right) v+\left(\left(v-l-r y^{\perp}\right) \cdot \nabla\right) v^{2} & \\
+r^{1} v^{\perp}+r v^{2 \perp}+\nabla q=0, & \text { in }[0, T] \times \Omega, \\
\operatorname{div} v=0, & \text { in }[0, T] \times \Omega, \\
v \cdot n=\left(l+r y^{\perp}\right) \cdot n, & \text { on }[0, T] \times \partial \Omega, \\
\lim _{|y| \rightarrow \infty} v(t, y)=0, & \text { in }[0, T], \\
m i=\int_{\partial \Omega}^{q n d \sigma-m\left(r^{1} l^{\perp}+r l^{2 \perp}\right),} & \text { in }[0, T], \\
J \dot{r}=\int_{\partial \Omega} q n \cdot y^{\perp} d \sigma, & \text { in }[0, T], \\
v(0, y)=0, & \forall y \in \Omega, \\
l(0)=0, \quad r(0)=0 . &
\end{aligned}
$$

In order to prove that $(v, l, r)=(0,0,0)$, we establish some energy estimate for (3.56)(3.63).

Multiplying (3.56) by $v$ and integrating over $(0, t) \times \Omega$, we obtain that

$$
\begin{aligned}
0=\int_{0}^{t} \int_{\Omega} v_{t} \cdot v d y & d s+\int_{0}^{t} \int_{\Omega}\left(\left(v^{1}-l^{1}-r^{1} y^{\perp}\right) \cdot \nabla\right) v \cdot v d y d s+\int_{0}^{t} \int_{\Omega}\left(\left(v-l-r y^{\perp}\right) \cdot \nabla\right) v^{2} \cdot v d y d s \\
& +\int_{0}^{t} \int_{\Omega} r v^{2 \perp} \cdot v d y d s+\int_{0}^{t} \int_{\Omega} \nabla q \cdot v d y d s=I_{1}+I_{2}+I_{3}+I_{4}+I_{5} .
\end{aligned}
$$

We now study each integral term. We easily have that

$$
I_{1}=\frac{1}{2} \int_{\Omega}|v(t)|^{2} \mathrm{~d} y \text {. }
$$

Next, some integrations by part give that

$$
I_{2}=\int_{0}^{t} \int_{\partial \Omega}\left(\sum_{j=1}^{m} w_{j} \chi_{j}\right) \frac{|v|^{2}}{2} d \sigma d s \leqslant C\|w\|_{C^{1}([0, T])} \int_{0}^{t}\|v\|_{H^{1}\left(\Omega_{R}\right)}^{2} d s
$$

where $\Omega_{R}=\{y \in \Omega ;|y|<R\}$ and $R$ is chosen so that $R>1+\sup _{y \in \partial \Omega}|y|$. Note that $v$ solves

$$
\begin{aligned}
\operatorname{curl} v & =\omega \\
\operatorname{div} v & =0 \\
v \cdot n & =\left(l+r y^{\perp}\right) \cdot n, \\
\int_{\partial \Omega} v(t, y) \cdot \tau d \sigma & =-\int_{\Omega} \omega(t, y) d y \\
\lim _{|y| \rightarrow \infty} v(t, y) & =0 .
\end{aligned}
$$


Pick a cutoff function $\kappa \in C_{0}^{\infty}\left(\mathbb{R}^{2}\right)$ with $\kappa(y)=1$ for $|y|<R, \kappa(y)=0$ for $|y|>R+1$.

Note that $v=\kappa v$ on $\Omega_{R}$, and that

$$
\begin{aligned}
\operatorname{curl}(\kappa v) & =-\nabla \kappa \cdot v^{\perp}+\kappa \omega, \\
\operatorname{div}(\kappa v) & =\nabla \kappa \cdot v, \\
\kappa v \cdot n & = \begin{cases}\left(l+r y^{\perp}\right) \cdot n & \text { for } y \in \partial \Omega, \\
0 & \text { for }|y|=R+1 .\end{cases}
\end{aligned}
$$

It follows from standard elliptic estimates that

$$
\begin{aligned}
\|v\|_{H^{1}\left(\Omega_{R}\right)} & \leqslant\|\kappa v\|_{H^{1}\left(\Omega_{R+1}\right)} \\
& \leqslant C\left(\|v\|_{L^{2}\left(\Omega_{R+1}\right)}+\|\omega\|_{L^{2}\left(\Omega_{R+1}\right)}+|l|+|r|\right) .
\end{aligned}
$$

Therefore

$$
\left|I_{2}\right| \leqslant C \int_{0}^{t}\left(|l(s)|^{2}+|r(s)|^{2}+\|v(s)\|_{L^{2}(\Omega)}^{2}+\|\omega(s)\|_{L^{2}\left(\Omega_{R+1}\right)}^{2}\right) d s .
$$

Let $\Phi=\Phi^{1}-\Phi^{2}$. Then

$$
\begin{array}{r}
\frac{\partial \Phi}{\partial t}=\left(\pi v^{1}\right)\left(t, \Phi^{1}(t, s, y)\right)-\left(\pi v^{1}\right)\left(t, \Phi^{2}(t, s, y)\right)+(\pi v)\left(t, \Phi^{2}(t, s, y)\right) \\
-l(t)-\left(r^{1}(t) \Phi(t, s, y)^{\perp}+r(t) \Phi^{2}(t, s, y)^{\perp}\right)
\end{array}
$$

with $\Phi(s, s, y)=0$.

Since $\pi\left(v^{1}\right) \in L^{\infty}\left(0, T ; W^{1, \infty}\left(\mathbb{R}^{2}\right)\right)$, this gives for $0 \leqslant t \leqslant s \leqslant T$ and $|y|<R$ that

$$
\left|\frac{\partial|\Phi|^{2}}{\partial t}\right| \leqslant C\left(|\Phi(t, s, y)|^{2}+\left|(\pi v)\left(t, \Phi^{2}(t, s, y)\right)\right|^{2}+|l(t)|^{2}+|r(t)|^{2}\right) .
$$

Thus, with Gronwall Lemma,

$$
|\Phi(0, s, y)|^{2} \leqslant C \int_{0}^{s}\left(\left|(\pi v)\left(\tau, \Phi^{2}(\tau, s, y)\right)\right|^{2}+|l(\tau)|^{2}+|r(\tau)|^{2}\right) d \tau
$$

Therefore

$$
\begin{aligned}
\int_{0}^{t}\|\omega(s)\|_{L^{2}\left(\Omega_{R+1}\right)}^{2} d s & \leqslant \int_{0}^{t} \int_{\Omega_{R+1}}\left|\pi\left(\omega_{0}\right)\left(\Phi^{1}(0, s, y)\right)-\pi\left(\omega_{0}\right)\left(\Phi^{2}(0, s, y)\right)\right|^{2} d y d s \\
& \leqslant C \int_{0}^{t} \int_{\Omega_{R+1}}|| \pi\left(\omega_{0}\right) \|_{W^{1, \infty}\left(\mathbb{R}^{2}\right)}^{2} \int_{0}^{s}\left(\left|\pi(v)\left(\tau, \Phi^{2}(\tau, s, y)\right)\right|^{2}+|l(\tau)|^{2}+|r(\tau)|^{2}\right) d \tau d y d s \\
& \left.\leqslant C \int_{0}^{t} \int_{0}^{s}[\| \pi(v)(\tau)) \|_{L^{2}\left(\mathbb{R}^{2}\right)}^{2}+|l(\tau)|^{2}+|r(\tau)|^{2}\right] d \tau d s \\
& \leqslant C \int_{0}^{t}(t-s)\left(\|v(s)\|_{L^{2}(\Omega)}^{2}+|l(s)|^{2}+|r(s)|^{2}\right) d s
\end{aligned}
$$

Combining (3.64) to (3.65), we infer that

$$
\left|I_{2}\right| \leqslant C \int_{0}^{t}\left(|l(s)|^{2}+|r(s)|^{2}+\|v(s)\|_{L^{2}(\Omega)}^{2}\right) d s
$$

On the other hand, we have that

$$
\begin{aligned}
I_{3} & =\int_{0}^{t} \int_{\Omega}(v \cdot \nabla) v^{2} \cdot v \mathrm{~d} y d s-\int_{0}^{t} \int_{\Omega}(l \cdot \nabla) v^{2} \cdot v \mathrm{~d} y d s-\int_{0}^{t} \int_{\Omega}\left(r y^{\perp} \cdot \nabla\right) v^{2} \cdot v \mathrm{~d} y d s \\
& =I_{31}+I_{32}+I_{33} .
\end{aligned}
$$


We can estimate each part:

$$
\begin{gathered}
\left|I_{31}\right| \leqslant\left\|\nabla v^{2}\right\|_{L^{\infty}\left(Q_{T}\right)} \int_{0}^{t} \int_{\Omega}|v|^{2} \mathrm{~d} y d s \\
\left|I_{32}\right| \leqslant \int_{0}^{t}|l(s)|\left(\int_{\Omega}\left|\nabla v^{2}\right|^{2} \mathrm{~d} y\right)^{1 / 2}\left(\int_{\Omega}|v|^{2} \mathrm{~d} y\right)^{1 / 2} d s \\
\leqslant \frac{1}{2}\left\|v^{2}\right\|_{L^{\infty}\left(0, T ; H^{1}(\Omega)\right)}\left[\int_{0}^{t}\left(\int_{\Omega}|v|^{2} \mathrm{~d} y+|l|^{2}\right) d s\right] .
\end{gathered}
$$

and

$$
\begin{gathered}
\left|I_{33}\right| \leqslant \int_{0}^{t}|r(s)|\left(\int_{\Omega}|y|^{2}\left|\nabla v^{2}\right|^{2} \mathrm{~d} y\right)^{1 / 2}\left(\int_{\Omega}|v|^{2} \mathrm{~d} y\right)^{1 / 2} d s \\
\leqslant \frac{1}{2}\left\||y| \nabla v^{2}\right\|_{L^{\infty}\left(0, T ; L^{2}(\Omega)\right)}\left[\int_{0}^{t}\left(\int_{\Omega}|v|^{2} \mathrm{~d} y+|r|^{2}\right) d s\right] .
\end{gathered}
$$

On the other hand,

$$
\begin{gathered}
\left|I_{4}\right| \leqslant \int_{0}^{t}|r(s)|\left(\int_{\Omega}\left|v^{2}\right|^{2} \mathrm{~d} y\right)^{1 / 2}\left(\int_{\Omega}|v|^{2} \mathrm{~d} y\right)^{1 / 2} d s \\
\leqslant \frac{1}{2}\left\|v^{2}\right\|_{L^{\infty}\left(0, T ; L^{2}(\Omega)\right)}\left[\int_{0}^{t}\left(\int_{\Omega}|v|^{2} \mathrm{~d} y+|r|^{2}\right) d s\right] .
\end{gathered}
$$

Finally we have that

$$
\begin{aligned}
I_{5} & =\int_{0}^{t} \int_{\partial \Omega} q\left(l+r y^{\perp}\right) \cdot n d \sigma d s \\
& =\frac{m}{2}|l(t)|^{2}+\frac{J}{2}|r(t)|^{2}+m \int_{0}^{t} l \cdot\left(r l^{2 \perp}\right) d s \\
& =I_{51}+I_{52}+I_{53}
\end{aligned}
$$

with

$$
\left|I_{53}\right| \leqslant \frac{m}{2}\left\|l^{2}\right\|_{L^{\infty}(0, T)} \int_{0}^{t}\left(|l|^{2}+|r|^{2}\right) d s .
$$

Thus, we have that

$$
\int_{\Omega}|v(t)|^{2} \mathrm{~d} y+m|l(t)|^{2}+J|r(t)|^{2} \leqslant C\left[\int_{0}^{t}\left(\int_{\Omega}|v|^{2} \mathrm{~d} y+m|l|^{2}+J|r|^{2}\right) d s\right]
$$

which gives with Gronwall's Lemma,

$$
v=0 \quad \text { in } \quad(0, T) \times \Omega \quad \text { and } \quad(l, r)=(0,0) \text { in }(0, T) .
$$

Using (3.56) we conclude that $\nabla q=0$ in $(0, T) \times \Omega$. We have proved the uniqueness of $(v, q, l, r)$ in the class (3.13)-(3.15). Let us show now that the map $w \in H^{2}(0, T) \rightarrow(l, r) \in$ $C([0, T])$ is continuous. Assume that $w^{k} \rightarrow w$ in $H^{2}(0, T)$, and let $\left(v^{k}, q^{k}, l^{k}, r^{k}\right)$ denote the solution of (3.1)-(3.8) and (3.53) associated with the initial data $\left(v_{0}, l_{0}, r_{0}\right)$ and the control $w^{k}$. Since

$$
\left\|w^{k}\right\|_{C^{1}([0, T])} \leqslant C\left\|w^{k}\right\|_{H^{2}(0, T)} \leqslant C
$$


we infer from the bootstrap argument in the proof of Proposition 3.1 that

$$
\begin{array}{rll}
\omega^{k} & \text { is bounded in } & L^{\infty}\left(0, T ; L_{\theta}^{1}(\Omega) \cap C^{k, \alpha}(\bar{\Omega})\right), \\
v^{k} & \text { is bounded in } & L^{\infty}\left(0, T ; C^{k+1, \alpha}(\bar{\Omega})\right) \cap H^{1}\left(0, T ; L^{2}(\Omega)\right), \\
|y| \nabla v^{k} & \text { is bounded in } & L^{\infty}\left(0, T ; L^{2}(\Omega)\right), \\
\nabla q^{k} & \text { is bounded in } & L^{\infty}\left(0, T ; L^{2}(\Omega)\right), \\
\text { and that }\left(l^{k}, r^{k}\right) & \text { is bounded in } & W^{1, \infty}(0, T) .
\end{array}
$$

Extracting subsequences, we can assume that

$$
w^{k} \rightarrow w \quad \text { in } C^{1}([0, T]),
$$

and that for some functions $l, r, \omega$

$$
\begin{aligned}
\left(l^{k}, r^{k}\right) & \rightarrow(l, r) \quad \text { in } C([0, T]), \\
\omega^{k} & \rightarrow \omega \quad \text { in } C\left([0, T] ; L_{\theta^{\prime}}^{1}(\Omega) \cap C^{k, \alpha^{\prime}}(\bar{\Omega})\right),
\end{aligned}
$$

for all $2<\theta^{\prime}<\theta$ and all $0<\alpha^{\prime}<\alpha$. This yields for some fonctions $v, q$ that

$$
\begin{array}{rlll}
v^{k} & \rightarrow & v & \text { in } C\left([0, T] ; C^{k+1, \alpha^{\prime}}(\bar{\Omega}) \cap W^{1, p}(\Omega)\right), \quad \text { for all } p \in(2, \infty), \\
v^{k} & \stackrel{w}{\longrightarrow} & v \quad \text { in } H^{1}\left(0, T ; L^{2}(\Omega)\right), \\
|y| \nabla v^{k} & \stackrel{w *}{\longrightarrow}|y| \nabla v \text { in } L^{\infty}\left(0, T ; L^{2}(\Omega)\right), \\
\nabla q^{k} & \stackrel{w *}{\longrightarrow} \quad \nabla q \quad \text { in } L^{\infty}\left(0, T ; L^{2}(\Omega)\right) .
\end{array}
$$

We can therefore pass to the limit in (3.1)-(3.8). We also notice that if $\Phi^{k}$ (resp. $\Phi$ ) denotes the flow associated with $\pi\left(v^{k}\right)-l^{k}-r^{k} y^{\perp}$ (resp. with $\left.\pi(v)-l-r y^{\perp}\right)$, then

$$
\omega^{k}(t, y)=\pi\left(\omega_{0}\right)\left(\Phi^{k}(0, t, y)\right) \rightarrow \pi\left(\omega_{0}\right)(\Phi(0, t, y))
$$

pointwise. Thus (3.53) holds. We conclude that $(v, q, l, r)$ is the unique solution of (3.1) -(3.8) and (3.53) associated with the data $\left(v_{0}, l_{0}, r_{0}\right)$ and the control $w$ in the class (3.13)(3.15) (with $\alpha$ replaced by $\alpha^{\prime}$ ). The proof of Proposition 3.5 is achieved.

\section{MAIN RESUlT}

From now on, the pressure will be denoted by $\mathbf{q}$. It should not be confused with the state vector $q=\left(h_{1}, h_{2}, \theta\right)$. We are now in a position to state and prove the main result in this paper.

Theorem 4.1. Assume that the rank conditions (2.42) and (2.43) are fulfilled, and pick any $T_{0}>0$. Then there exists $\eta>0$ such that for any $\left(h_{0}, \theta_{0}, l_{0}, r_{0}\right) \in \mathbb{R}^{6}$ and any $\left(h_{T}, \theta_{T}, l_{T}, r_{T}\right) \in \mathbb{R}^{6}$ with

$$
\left|\left(h_{0}, \theta_{0}\right)\right|<\eta, \quad\left|\left(h_{T}, \theta_{T}\right)\right|<\eta,
$$

and for any $\omega_{0} \in W^{1, \infty}(\Omega) \cap L_{\theta}^{1}(\Omega)$ with $\theta>2$, if $v_{0}$ denotes the solution of (3.12) with $w_{j}(0)=0$ for $1 \leqslant j \leqslant m$, then there exist a time $T \in\left(0, T_{0}\right]$ and a control input $w \in H^{2}\left(0, T, \mathbb{R}^{2}\right)$ with $w(0)=0$ such that the system (3.1)-(3.11) admits a solution $(h, \theta, l, r, v, \mathbf{q})$ satisfying

$$
(h, \theta, l, r)_{\mid t=T}=\left(h_{T}, \theta_{T}, l_{T}, r_{T}\right) .
$$

Proof. Let $\omega_{0} \in W^{1, \infty}(\Omega) \cap L_{\theta}^{1}(\Omega)$, and write $\left(q_{0}, p_{0}\right)=\left(h_{0}, \theta_{0}, l_{0}, r_{0}\right),\left(q_{T}, p_{T}\right)=\left(h_{T}, \theta_{T}, l_{T}, r_{T}\right)$. The proof is done in two steps. In a first step, we prove the result for $\left\|\omega_{0}\right\|_{C^{0, \alpha}},\left\|\omega_{0}\right\|_{L_{\theta}^{1}}$, $\left|l_{0}\right|,\left|r_{0}\right|,\left|l_{T}\right|$ and $\left|r_{T}\right|$ small enough, and in a second step, we remove that assumption by performing a scaling in time. 
STEP 1. Let $w$ be as in (2.68) for $T=T_{0}$. We may pick a number $\eta_{1}>0$ such that $w\left(q_{0}, p_{0}, q_{T}, p_{T}\right)$ is defined for $\left|\left(q_{0}, p_{0}\right)\right| \leqslant \eta_{1}$ and $\left|\left(q_{T}, p_{T}\right)\right| \leqslant \eta_{1}$, with $\|w\|_{C^{1}([0, T])} \leqslant 1$. Pick any initial state $\left(q_{0}, p_{0}\right)=\left(h_{0}, \theta_{0}, l_{0}, r_{0}\right)$ with $\left|\left(q_{0}, p_{0}\right)\right| \leqslant \eta_{1}$. For any given $\left(q_{T}, p_{T}, v_{0}\right)$ we denote by $(h, \theta, l, r, v, \mathbf{q})$ the solution of (3.1)-(3.11) and (3.53) corresponding to the velocity $v_{0}$ and to the control $w=w\left(q_{0}, p_{0}, q_{T}, p_{T}\right)$ (see $(2.68)$ ), and by $(\bar{h}, \bar{\theta}, \bar{l}, \bar{r}, \bar{v}, \overline{\mathbf{q}})$ the solution corresponding to $\left(q_{0}, p_{0}\right)$ together with the velocity $\bar{v}_{0}$ which solves

$$
\begin{gathered}
\operatorname{curl} \bar{v}_{0}=0, \\
\operatorname{div} \bar{v}_{0}=0, \\
\bar{v}_{0} \cdot n=\left(l_{0}+r_{0} y^{\perp}\right) \cdot n, \\
\int_{\partial \Omega} \bar{v}_{0} \cdot \tau d \sigma=0, \\
\lim _{|y| \rightarrow \infty} \bar{v}_{0}(y)=0
\end{gathered}
$$

and the (same) control $w$. Pick any $\alpha \in(0,1)$. Obviously, $W^{1, \infty}(\Omega) \subset C^{0, \alpha}(\bar{\Omega})$. From (3.16) we infer that there exists some constant $C_{1}>0$ such that

$$
\|(l-\bar{l}, r-\bar{r})\|_{L^{\infty}(0, T)} \leqslant C_{1}\left(\left\|\omega_{0}\right\|_{C^{0, \alpha}(\bar{\Omega})}+\left\|\omega_{0}\right\|_{L_{\theta}^{1}(\Omega)}\right)
$$

whenever

$$
\left|\left(l_{0}, r_{0}\right)\right| \leqslant 1, \quad\left\|\omega_{0}\right\|_{C^{0, \alpha}(\bar{\Omega})}+\left\|\omega_{0}\right\|_{L_{\theta}^{1}(\Omega)} \leqslant 1, \quad \text { and }\|w\|_{C^{1}([0, T])} \leqslant 1 .
$$

Combined to the equations

$$
\begin{array}{ll}
\dot{\bar{h}}=Q(\bar{\theta}) \bar{l}, & \dot{\bar{\theta}}=\bar{r}, \\
\dot{h}=Q(\theta) l, & \dot{\theta}=r,
\end{array}
$$

this gives for some constant $C_{2}>0$

$$
\|(h-\bar{h}, \theta-\bar{\theta})\|_{L^{\infty}(0, T)} \leqslant C_{2}\left(\left\|\omega_{0}\right\|_{C^{0, \alpha}(\bar{\Omega})}+\left\|\omega_{0}\right\|_{L_{\theta}^{1}(\Omega)}\right),
$$

provided that (4.2) holds. Let $f: \bar{B}=\left\{x \in \mathbb{R}^{6} ;|x| \leqslant 1\right\} \rightarrow \mathbb{R}^{6}$ be defined by

$$
f\left(x_{T}\right)=\eta_{1}^{-1}(q(T), p(T))
$$

where $\left(q_{T}, p_{T}\right)=: \eta_{1} x_{T}$.

We notice that $f$ is continuous, by virtue of Proposition 3.5 and (3.9)-(3.11). Pick any $\varepsilon \in(0,1)$. From (4.1) and (4.3), we deduce that for

$$
\begin{aligned}
& \left|\left(l_{0}, r_{0}\right)\right| \leqslant 1, \\
& || \omega_{0}\left\|_{C^{0, \alpha}(\bar{\Omega})}+\right\| \omega_{0} \|_{L_{\theta}^{1}(\Omega)}<\delta,
\end{aligned}
$$

with $\delta$ small enough, we have that

$$
\left|f\left(x_{T}\right)-x_{T}\right|<\varepsilon, \quad \text { for }\left|x_{T}\right| \leqslant 1 .
$$

We need the following topological result.

Lemma 4.2. Let $B=\left\{x \in \mathbb{R}^{n} ;|x|<1\right\}$ and $S=\partial B$. Let $f: \bar{B} \rightarrow \mathbb{R}^{n}$ be a continuous map such that for some constant $\varepsilon \in(0,1)$

$$
|f(x)-x| \leqslant \varepsilon \quad \forall x \in S .
$$

Then

$$
(1-\varepsilon) B \subset f(\bar{B})
$$


Proof. We shall use classical results from degree theory (see e.g. [7, Appendix B] or [17]). Assume that (4.7) is not true. Then there exists $x^{*} \in \mathbb{R}^{n}$ with

$$
\left|x^{*}\right|<1-\varepsilon \text { and } x^{*} \neq f(x) \forall x \in \bar{B} \text {. }
$$

Let us introduce the continuous map $H:[0,1] \times \bar{B} \rightarrow \mathbb{R}^{n}$ defined by

$$
H(s, x)= \begin{cases}f(2 s x)-x^{*} & \text { if } 0 \leqslant s \leqslant \frac{1}{2}, x \in \bar{B} \\ (2 s-1) x+2(1-s) f(x)-x^{*} & \text { if } \frac{1}{2} \leqslant s \leqslant 1, x \in \bar{B} .\end{cases}
$$

We claim that $H(s, x) \neq 0$ for all $(s, x) \in[0,1] \times S$. Indeed, for any $(s, x) \in[0,1 / 2] \times S$, we have $f(2 s x) \neq x^{*}$ by (4.8). On the other hand, for any given $(s, x) \in[1 / 2,1] \times S$, we have

$$
\begin{aligned}
\left|(2 s-1) x+2(1-s) f(x)-x^{*}\right| & \geqslant|x+2(1-s)(f(x)-x)|-\left|x^{*}\right| \\
& >1-2(1-s) \varepsilon-(1-\varepsilon) \geqslant 0
\end{aligned}
$$

which yields the result. It follows from the homotopy invariance of the degree (see e.g. [7, Proposition B.8]) that

$$
\operatorname{deg}\left(f(0)-x^{*}, B, 0\right)=\operatorname{deg}\left(x-x^{*}, B, 0\right) .
$$

This yields a contradiction, since $\operatorname{deg}\left(f(0)-x^{*}, B, 0\right)=0$ by [7, Proposition B.10] while $\operatorname{deg}\left(x-x^{*}, B, 0\right)=1$ by [7, (B.4) p. 380].

Thus, we infer from Lemma 4.2 that if $\left(q_{0}, p_{0}, q_{T}, p_{T}\right) \in \mathbb{R}^{12}$ is such that

$$
\left|\left(q_{0}, p_{0}\right)\right|<\eta_{1}, \quad\left|\left(q_{T}, p_{T}\right)\right|<\eta_{2}:=\eta_{1}(1-\varepsilon),
$$

and (4.4)-(4.5) are satisfied, then there exists a control $w=w\left(q_{0}, p_{0}, \tilde{q}_{T}, \tilde{p}_{T}\right)$ for which the solution of (3.1)-(3.11) satisfies $(h(T), \theta(T), l(T), r(T))=(q(T), p(T))=\left(q_{T}, p_{T}\right)$.

STEP 2. To drop the assumptions (4.4)-(4.5) (corresponding to a given time $T_{0}>0$ ), we use a scaling in time introduced in [6] for the control of Euler equations. Let $\left(q_{0}, p_{0}\right)$, $\left(q_{T}, p_{T}\right)$, and $v_{0}$ be given data with

$$
\left|q_{0}\right|<\eta_{2}, \quad\left|q_{T}\right|<\eta_{2} .
$$

We set $p_{0}^{\lambda}=\lambda p_{0}, p_{T}^{\lambda}=\lambda p_{T}$, and $v_{0}^{\lambda}=\lambda v_{0}$. Then for $\lambda>0$ small enough, we have that

$$
\left|\left(q_{0}, p_{0}^{\lambda}\right)\right|<\eta_{2}, \quad\left|\left(q_{T}, p_{T}^{\lambda}\right)\right|<\eta_{2}, \quad\left|p_{0}^{\lambda}\right| \leqslant 1,
$$

and $\omega_{0}^{\lambda}:=\operatorname{curl} v_{0}^{\lambda}$ satisfies

$$
\left\|\omega_{0}^{\lambda}\right\|_{C^{0, \alpha}(\bar{\Omega})}+\left\|\omega_{0}^{\lambda}\right\|_{L_{\theta}^{1}(\Omega)}<\delta .
$$

By Step 1 , there exists some trajectory $\left(q^{\lambda}, p^{\lambda}\right)$ for the boat connecting $\left(q_{0}, p_{0}^{\lambda}\right)$ at $t=0$ to $\left(q_{T}, p_{T}^{\lambda}\right)$ at $t=T_{0}$, with corresponding fluid velocity $v^{\lambda}$, pressure $\mathbf{q}^{\lambda}$, and control $w^{\lambda}$. Let us set

$$
\begin{aligned}
q(t) & =q^{\lambda}\left(\lambda^{-1} t\right), \\
p(t) & =\lambda^{-1} p^{\lambda}\left(\lambda^{-1} t\right), \\
v(t, y) & =\lambda^{-1} v^{\lambda}\left(\lambda^{-1} t, y\right), \\
\mathbf{q}(t, y) & =\lambda^{-2} \mathbf{q}^{\lambda}\left(\lambda^{-1} t, y\right), \\
w(t) & =\lambda^{-1} w^{\lambda}\left(\lambda^{-1} t\right),
\end{aligned}
$$

for $y \in \Omega$ and $0 \leqslant t \leqslant T:=\lambda T_{0}$. Then $(q, p)$ is a trajectory for the boat connecting $\left(q_{0}, p_{0}\right)$ at $t=0$ to $\left(q_{T}, p_{T}\right)$ at $t=T$ and corresponding to the initial fluid velocity $v_{0}$. 


\section{Appendix}

\subsection{Proof of Proposition 2.1.}

Applying the operator curl in (1.18) yields

$$
\omega_{t}+\left(v-l-r y^{\perp}\right) \cdot \nabla \omega=0 .
$$

Let $\tilde{v}(t, y):=v(t, y)-l(t)-r(t) y^{\perp}$ and let $\varphi=\varphi(t, s, y)$ denote the flow associated with $\tilde{v}$, i.e.

$$
\frac{\partial \varphi}{\partial t}=\tilde{v}(t, \varphi), \quad \varphi_{\mid t=s}=y .
$$

Following Yudovich [11], we introduce the time $t^{*}(t, y)$ at which the fluid element first appears in the domain, and set $y^{*}(t, y)=\varphi\left(t^{*}(t, y), t, y\right)$. Then either $t^{*}=0$, or $t^{*}>0$ and $y^{*} \in \partial \Omega$ with $\sum_{j=1}^{m} w_{j}\left(t^{*}\right) \chi_{j}\left(y^{*}\right)<0$. Integrating in (5.1) yields

$$
\omega(t, y)=\omega\left(t^{*}, y^{*}\right)
$$

which, combined to (2.1) and (2.3), gives (2.4). For (2.5), we compute the time-derivative of the circulation using (1.18)

$$
\begin{aligned}
\frac{d}{d t} \int_{\partial \Omega} v(t, y) \cdot \tau d \sigma & =\int_{\partial \Omega} v_{t} \cdot \tau d \sigma \\
& =-\int_{\partial \Omega}\left[\left(v-l-r y^{\perp}\right) \cdot \nabla v\right] \cdot \tau d \sigma-r \int_{\partial \Omega} v^{\perp} \cdot \tau d \sigma
\end{aligned}
$$

As

$$
\operatorname{curl}\left[\left(v-l-r y^{\perp}\right) \cdot \nabla v\right]=\left(v-l-r y^{\perp}\right) \cdot \nabla \omega=0,
$$

we obtain by Stokes' theorem

$$
\int_{\partial \Omega}\left[\left(v-l-r y^{\perp}\right) \cdot \nabla v\right] \cdot \tau d \sigma=0
$$

provided that $\left(v-l-r y^{\perp}\right) \cdot \nabla v \in L^{2}(\Omega) \cap C^{1}(\bar{\Omega})$ (see [19, Lemma 2.5]). On the other hand,

$$
\int_{\partial \Omega} v^{\perp} \cdot \tau d \sigma=-\int_{\partial \Omega} v \cdot n d \sigma=-\int_{\partial \Omega}\left(l+r y^{\perp}\right) \cdot n-\sum_{1 \leqslant j \leqslant m} w_{j}(t) \int_{\partial \Omega} \chi_{j}(y) d \sigma=0 .
$$

This completes the proof of $(2.5)$.

\subsection{Proof of Proposition 2.4.}

We first express the pressure $q$ in terms of $l, r, v$ and their derivatives. Using (2.4), we easily obtain

$$
v \cdot \nabla v=\nabla \frac{|v|^{2}}{2} \quad \text { and }-r y^{\perp} \cdot \nabla v+r v^{\perp}=r \nabla\left(y \cdot v^{\perp}\right)
$$

Thus (1.18) gives

$$
\begin{aligned}
-\nabla q & =\frac{\partial v}{\partial t}+\nabla\left(\frac{|v|^{2}}{2}-l \cdot v-r y^{\perp} \cdot v\right) \\
& =\nabla\left(\sum_{1 \leqslant i \leqslant 2} i_{i} \Phi_{i}+\dot{r} \Phi_{3}+\sum_{1 \leqslant j \leqslant m} \dot{w}_{j} \Psi_{j}+\frac{|v|^{2}}{2}-l \cdot v-r y^{\perp} \cdot v\right)
\end{aligned}
$$


hence we can take

$$
q=-\left\{\sum_{1 \leqslant i \leqslant 2} \dot{l}_{i} \Phi_{i}+\dot{r} \Phi_{3}+\sum_{1 \leqslant j \leqslant m} \dot{w}_{j} \Psi_{j}+\frac{|v|^{2}}{2}-l \cdot v-r y^{\perp} \cdot v\right\} .
$$

Replacing $q$ by its value in (1.22) yields

$$
\begin{aligned}
m \dot{l}= & \int_{\partial \Omega} q n d \sigma-m r l^{\perp} \\
= & -m r l^{\perp}-\left\{\sum_{1 \leqslant i \leqslant 2} i_{i} \int_{\partial \Omega} \Phi_{i} n d \sigma+\dot{r} \int_{\partial \Omega} \Phi_{3} n d \sigma+\sum_{1 \leqslant j \leqslant m} \dot{w}_{j} \int_{\partial \Omega} \Psi_{j} n d \sigma\right. \\
& \left.+\int_{\partial \Omega}\left(\frac{|v|^{2}}{2}-\left(l+r y^{\perp}\right) \cdot v\right) n d \sigma\right\} .
\end{aligned}
$$

Using (5.4), (1.19)-(1.20) we obtain

$$
\begin{aligned}
\int_{\partial \Omega} \frac{|v|^{2}}{2} n d \sigma & =\int_{\Omega} \nabla \frac{|v|^{2}}{2} d y \\
& =\int_{\Omega} v \cdot \nabla v d y \\
& =-\int_{\Omega}(\operatorname{div} v) v d y+\int_{\partial \Omega}(v \cdot n) v d \sigma \\
& =\int_{\partial \Omega}\left[\left(l+r y^{\perp}\right) \cdot n\right] v d \sigma+\int_{\partial \Omega}\left(\sum_{1 \leqslant j \leqslant m} w_{j}(t) \chi_{j}(y)\right) v d \sigma .
\end{aligned}
$$

Using the following identity

$$
(w \cdot n) v-(w \cdot v) n=-(v \cdot \tau) w^{\perp} \quad \forall v, w \in \mathbb{R}^{2},
$$

we obtain that

$$
\int_{\partial \Omega}\left[\left(l+r y^{\perp}\right) \cdot n v-\left(l+r y^{\perp}\right) \cdot v n\right] d \sigma=-\int_{\partial \Omega}(v \cdot \tau)\left(l^{\perp}-r y\right) d \sigma
$$

hence, using (2.5),

$$
\int_{\partial \Omega}\left(\frac{|v|^{2}}{2}-\left(l+r y^{\perp}\right) \cdot v\right) n d \sigma=r \int_{\partial \Omega} y(v \cdot \tau) d \sigma+\int_{\partial \Omega}\left(\sum_{1 \leqslant j \leqslant m} w_{j}(t) \chi_{j}(y)\right) v d \sigma
$$

Therefore, from (5.6) and (5.8), we obtain

$$
\begin{aligned}
m i= & -m r l^{\perp}-\left\{\sum_{1 \leqslant i \leqslant 2} i_{i} \int_{\partial \Omega} \Phi_{i} n d \sigma+\dot{r} \int_{\partial \Omega} \Phi_{3} n d \sigma+\sum_{1 \leqslant j \leqslant m} \dot{w}_{j} \int_{\partial \Omega} \Psi_{j} n d \sigma\right. \\
& \left.+r \int_{\partial \Omega} y(v \cdot \tau) d \sigma+\int_{\partial \Omega}\left(\sum_{1 \leqslant j \leqslant m} w_{j} \chi_{j}\right) v d \sigma\right\} .
\end{aligned}
$$


Let us turn our attention to the dynamics of $r$. Substituting the expression of $q$ given in (5.5) in (1.23) yields

$$
\begin{aligned}
J \dot{r}=- & \left\{\sum_{1 \leqslant i \leqslant 2} i_{i} \int_{\partial \Omega} \Phi_{i} y^{\perp} \cdot n d \sigma+\dot{r} \int_{\partial \Omega} \Phi_{3} y^{\perp} \cdot n d \sigma+\sum_{1 \leqslant j \leqslant m} \dot{w}_{j} \int_{\partial \Omega} \Psi_{j} y^{\perp} \cdot n d \sigma\right. \\
& \left.+\int_{\partial \Omega}\left(\frac{|v|^{2}}{2}-\left(l+r y^{\perp}\right) \cdot v\right)\left(y^{\perp} \cdot n\right) d \sigma\right\} .
\end{aligned}
$$

Using (5.4) and the fact that $\operatorname{div}\left(y^{\perp}\right)=0$ we obtain

$$
\begin{aligned}
\int_{\partial \Omega} \frac{|v|^{2}}{2}\left(y^{\perp} \cdot n\right) d \sigma & =\int_{\Omega} \operatorname{div}\left(\frac{|v|^{2}}{2} y^{\perp}\right) d y \\
& =\int_{\Omega}(v \cdot \nabla v) \cdot y^{\perp} d y \\
& =\int_{\Omega}\left(\sum_{1 \leqslant i \leqslant 2} v_{i} \partial_{i} v\right) \cdot y^{\perp} d y \\
& =-\int_{\Omega} \sum_{1 \leqslant i \leqslant 2} v_{i} \partial_{i}\left(y^{\perp}\right) \cdot v d y+\int_{\partial \Omega}(v \cdot n)\left(v \cdot y^{\perp}\right) d \sigma
\end{aligned}
$$

But $\sum_{1 \leqslant i \leqslant 2} v_{i} \partial_{i} y^{\perp}=v^{\perp}$, hence

$$
\int_{\Omega} \sum_{1 \leqslant i \leqslant 2} v_{i} \partial_{i}\left(y^{\perp}\right) \cdot v d y=0
$$

and

$$
\begin{aligned}
\int_{\partial \Omega} & \left(\frac{|v|^{2}}{2}-\left(l+r y^{\perp}\right) \cdot v\right)\left(y^{\perp} \cdot n\right) d \sigma \\
\quad & \int_{\partial \Omega}\left[\left(l+r y^{\perp}\right) \cdot n\left(v \cdot y^{\perp}\right)-\left(l+r y^{\perp}\right) \cdot v\left(n \cdot y^{\perp}\right)\right] d \sigma+\int_{\partial \Omega}\left(\sum_{1 \leqslant j \leqslant m} w_{j} \chi_{j}\right)\left(v \cdot y^{\perp}\right) d \sigma \\
\quad & =-\int_{\partial \Omega}\left(l+r y^{\perp}\right)^{\perp} \cdot y^{\perp}(v \cdot \tau) d \sigma+\int_{\partial \Omega}\left(\sum_{1 \leqslant j \leqslant m} w_{j} \chi_{j}\right) v \cdot y^{\perp} d \sigma
\end{aligned}
$$

where we used again the identity (5.7). We conclude that

$$
\begin{aligned}
J \dot{r}=- & \left\{\sum_{1 \leqslant i \leqslant 2} i_{i} \int_{\partial \Omega} \Phi_{i} y^{\perp} \cdot n d \sigma+\dot{r} \int_{\partial \Omega} \Phi_{3} y^{\perp} \cdot n d \sigma+\sum_{1 \leqslant j \leqslant m} \dot{w}_{j} \int_{\partial \Omega} \Psi_{j} y^{\perp} \cdot n d \sigma\right. \\
& \left.-\int_{\partial \Omega}(l \cdot y)(v \cdot \tau) d \sigma+\int_{\partial \Omega}\left(\sum_{1 \leqslant j \leqslant m} w_{j} \chi_{j}\right) v \cdot y^{\perp} d \sigma\right\} .
\end{aligned}
$$

Before expanding the bilinear terms in (5.9)-(5.10), we exploit the symmetries in the shape of the rigid body and in the location of the control inputs in order to write only the nonvanishing terms in the final system. Recall that we have assumed that $S$ (hence also $\Omega)$ is symmetric with respect to the $y_{1}$-axis, i.e.

$$
\left(y_{1}, y_{2}\right) \in S \Rightarrow\left(y_{1},-y_{2}\right) \in S .
$$


Lemma 5.1. Let $\Omega, g$ and $\Psi$ be as in Lemma 2.2, with $s>1$ and $\lim _{|y| \rightarrow \infty} \Psi(y)=0$. Assume that for some $\varepsilon \in\{ \pm 1\}$, we have

$$
g\left(y_{1},-y_{2}\right)=\varepsilon g\left(y_{1}, y_{2}\right) \quad\left(y_{1}, y_{2}\right) \in \partial \Omega
$$

Then

$$
\Psi\left(y_{1},-y_{2}\right)=\varepsilon \Psi\left(y_{1}, y_{2}\right), \quad\left(y_{1}, y_{2}\right) \in \Omega
$$

and

$$
\int_{\partial \Omega} \Psi n d \sigma= \begin{cases}\left(\int_{\partial \Omega} \Psi n_{1} d \sigma, 0\right) & \text { if } \varepsilon=1 \\ \left(0, \int_{\partial \Omega} \Psi n_{2} d \sigma\right) & \text { if } \varepsilon=-1\end{cases}
$$

Proof. Let $\tilde{\Psi}\left(y_{1}, y_{2}\right)=\varepsilon \Psi\left(y_{1},-y_{2}\right)$ for $y=\left(y_{1}, y_{2}\right) \in \Omega$. Then $\tilde{\Psi} \in \widehat{H}^{s+1}(\Omega)$ and it fulfills $\Delta \tilde{\Psi}=0$ in $\Omega$ and $\lim _{|y| \rightarrow \infty} \tilde{\Psi}(y)=0$. On the other hand, for any $y \in \partial \Omega$

$$
\text { and } \quad \begin{aligned}
\nabla \tilde{\Psi}\left(y_{1}, y_{2}\right) & =\varepsilon\left(\partial_{1} \Psi\left(y_{1},-y_{2}\right),-\partial_{2} \Psi\left(y_{1},-y_{2}\right)\right) \\
n\left(y_{1}, y_{2}\right) & =\left(n_{1}\left(y_{1},-y_{2}\right),-n_{2}\left(y_{1},-y_{2}\right)\right) .
\end{aligned}
$$

Therefore

$$
\frac{\partial \tilde{\Psi}}{\partial n}\left(y_{1}, y_{2}\right)=(\nabla \tilde{\Psi} \cdot n)\left(y_{1}, y_{2}\right)=\varepsilon(\nabla \Psi \cdot n)\left(y_{1},-y_{2}\right)=\varepsilon g\left(y_{1},-y_{2}\right)=g\left(y_{1}, y_{2}\right)
$$

hence $\tilde{\Psi} \equiv \Psi$, i.e. (5.13) holds. To prove (5.14), we notice that when $\varepsilon=1$

$$
\left(\Psi n_{2}\right)\left(y_{1},-y_{2}\right)=-\left(\Psi n_{2}\right)\left(y_{1}, y_{2}\right) \quad \text { in } \partial \Omega
$$

hence $\int_{\partial \Omega} \Psi n_{2} d \sigma=0$. The proof of (5.14) when $\varepsilon=-1$ is similar.

Let $s$ denote the orthogonal symmetry with respect to the $y_{1}$-axis, and let

$$
y^{\prime}=s(y)=\left(y_{1},-y_{2}\right)
$$

be the point symmetric to $y=\left(y_{1}, y_{2}\right)$. Then

$$
n\left(y^{\prime}\right)=s(n(y)), y^{\perp}=-s\left(y^{\perp}\right) \quad \text { and } \quad n\left(y^{\prime}\right) \cdot y^{\prime \perp}=-n(y) \cdot y^{\perp} \quad \text { in } \partial \Omega .
$$

Recall that the functions $\chi_{j}$ fulfill the following symmetry properties

$$
\chi_{1}\left(y^{\prime}\right)=\chi_{1}(y), \quad \chi_{j}\left(y^{\prime}\right)=-\chi_{j}(y) \quad \text { for } j \geqslant 2 .
$$

An iterative application of Lemma 5.1 gives the following 
Proposition 5.2. $y^{\prime}$ and $s$ being as in (5.15), we have

$$
\begin{aligned}
& \Phi_{1}\left(y^{\prime}\right)=\Phi_{1}(y), \quad \nabla \Phi_{1}\left(y^{\prime}\right)=s\left[\nabla \Phi_{1}(y)\right] \\
& \int_{\partial \Omega} \Phi_{1} n d \sigma=\left(\int_{\partial \Omega} \Phi_{1} n_{1} d \sigma, 0\right), \quad \int_{\partial \Omega} \Phi_{1} y^{\perp} \cdot n d \sigma=0 ;
\end{aligned}
$$

(ii) $\quad \Phi_{2}\left(y^{\prime}\right)=-\Phi_{2}(y), \quad \nabla \Phi_{2}\left(y^{\prime}\right)=-s\left[\nabla \Phi_{2}(y)\right]$

$$
\int_{\partial \Omega} \Phi_{2} n d \sigma=\left(0, \int_{\partial \Omega} \Phi_{2} n_{2} d \sigma\right) \text {; }
$$

$$
\Phi_{3}\left(y^{\prime}\right)=-\Phi_{3}(y), \quad \nabla \Phi_{3}\left(y^{\prime}\right)=-s\left[\nabla \Phi_{3}(y)\right]
$$

$$
\int_{\partial \Omega} \Phi_{3} n d \sigma=\left(0, \int_{\partial \Omega} \Phi_{3} n_{2} d \sigma\right)
$$

(iv) $\quad \Psi_{1}\left(y^{\prime}\right)=\Psi_{1}(y), \quad \nabla \Psi_{1}\left(y^{\prime}\right)=s\left[\nabla \Psi_{1}(y)\right]$

$$
\int_{\partial \Omega} \Psi_{1} n d \sigma=\left(\int_{\partial \Omega} \Psi_{1} n_{1} d \sigma, 0\right), \quad \int_{\partial \Omega} \Psi_{1} y^{\perp} \cdot n d \sigma=0
$$

(v) $\quad$ For $2 \leqslant j \leqslant m, \Psi_{j}\left(y^{\prime}\right)=-\Psi_{j}(y), \quad \nabla \Psi_{j}\left(y^{\prime}\right)=-s\left[\nabla \Psi_{j}(y)\right]$

$$
\int_{\partial \Omega} \Psi_{j} n d \sigma=\left(0, \int_{\partial \Omega} \Psi_{j} n_{2} d \sigma\right) \text {. }
$$

Notice that $\int_{\partial \Omega} \Phi_{2} y^{\perp} \cdot n d \sigma, \int_{\partial \Omega} \Phi_{3} y^{\perp} \cdot n d \sigma$ and $\int_{\partial \Omega} \Psi_{j} y^{\perp} \cdot n d \sigma(j \geqslant 2)$ may be different from 0 .

(5.19), (5.21), (5.23), (5.25), and (5.27) will be used to simplify the linear terms in (5.9)-(5.10). Let us focus on the bilinear terms in (5.9)-(5.10). Let us begin with

$$
r \int_{\partial \Omega} y(v \cdot \tau) d \sigma=r \int_{\partial \Omega} y\left(\sum_{1 \leqslant i \leqslant 2} l_{i} \nabla \Phi_{i}+r \nabla \Phi_{3}+\sum_{1 \leqslant j \leqslant m} w_{j} \nabla \Psi_{j}\right) \cdot \tau d \sigma
$$

Since $\tau\left(y^{\prime}\right)=-s(\tau(y))$, we deduce from (5.18)-(5.26) that $\nabla \Phi_{1} \cdot \tau, \nabla \Psi_{1} \cdot \tau$ (resp. $\nabla \Phi_{2} \cdot \tau$, $\left.\nabla \Phi_{3} \cdot \tau, \nabla \Psi_{2} \cdot \tau\right)$ are odd (resp. even) functions with respect to the transformation $y \rightarrow y^{\prime}$. Therefore

$$
\begin{aligned}
& r \int_{\partial \Omega} y(v \cdot \tau) d \sigma \\
& \quad=r\left\{l_{1}\left(\begin{array}{c}
0 \\
\int_{\partial \Omega} y_{2} \nabla \Phi_{1} \cdot \tau d \sigma
\end{array}\right)+l_{2}\left(\begin{array}{c}
\int_{\partial \Omega} y_{1} \nabla \Phi_{2} \cdot \tau d \sigma \\
0
\end{array}\right)+r\left(\begin{array}{c}
\int_{\partial \Omega} y_{1} \nabla \Phi_{3} \cdot \tau d \sigma \\
0
\end{array}\right)\right. \\
& \left.\quad+w_{1}\left(\begin{array}{c}
0 \\
\int_{\partial \Omega} y_{2} \nabla \Psi_{1} \cdot \tau d \sigma
\end{array}\right)+\sum_{2 \leqslant j \leqslant m} w_{j}\left(\begin{array}{c}
\int_{\partial \Omega} y_{1} \nabla \Psi_{j} \cdot \tau d \sigma \\
0
\end{array}\right)\right\}
\end{aligned}
$$


On the other hand

$$
\begin{aligned}
& -\int_{\partial \Omega}(l \cdot y)(v \cdot \tau) d \sigma \\
& =-\int_{\partial \Omega}\left(l_{1} y_{1}+l_{2} y_{2}\right)\left(\sum_{1 \leqslant i \leqslant 2} l_{i} \nabla \Phi_{i}+r \nabla \Phi_{3}+\sum_{1 \leqslant j \leqslant m} w_{j} \nabla \Psi_{j}\right) \cdot \tau d \sigma \\
& =-l_{1}\left(l_{2} \int_{\partial \Omega} y_{1} \nabla \Phi_{2} \cdot \tau d \sigma+r \int_{\partial \Omega} y_{1} \nabla \Phi_{3} \cdot \tau d \sigma+\sum_{2 \leqslant j \leqslant m} w_{j} \int_{\partial \Omega} y_{1} \nabla \Psi_{j} \cdot \tau d \sigma\right) \\
& \quad-l_{2}\left(l_{1} \int_{\partial \Omega} y_{2} \nabla \Phi_{1} \cdot \tau d \sigma+w_{1} \int_{\partial \Omega} y_{2} \nabla \Psi_{1} \cdot \tau d \sigma\right) .
\end{aligned}
$$

Finally, using (5.17) and (5.18)-(5.27), we obtain

$$
\begin{aligned}
& \int_{\partial \Omega}\left(\sum_{1 \leqslant j \leqslant m} w_{j}(t) \chi_{j}(y)\right) v d \sigma \\
& =\sum_{1 \leqslant j \leqslant m} w_{j}(t)\left(\sum_{1 \leqslant i \leqslant 2} l_{i} \int_{\partial \Omega} \chi_{j} \nabla \Phi_{i} d \sigma+r \int_{\partial \Omega} \chi_{j} \nabla \Phi_{3} d \sigma+\sum_{1 \leqslant k \leqslant m} w_{k} \int_{\partial \Omega} \chi_{j} \nabla \Psi_{k} d \sigma\right) \\
& =w_{1}\left\{l_{1}\left(\begin{array}{c}
\int_{\partial \Omega} \chi_{1} \partial_{1} \Phi_{1} d \sigma \\
0
\end{array}\right)+l_{2}\left(\begin{array}{c}
0 \\
\int_{\partial \Omega} \chi_{1} \partial_{2} \Phi_{2} d \sigma
\end{array}\right)+r\left(\begin{array}{c}
0 \\
\int_{\partial \Omega} \chi_{1} \partial_{2} \Phi_{3} d \sigma
\end{array}\right)\right. \\
& \left.+w_{1}\left(\begin{array}{c}
\int_{\partial \Omega} \chi_{1} \partial_{1} \Psi_{1} d \sigma \\
0
\end{array}\right)+\sum_{2 \leqslant j \leqslant m} w_{j}\left(\begin{array}{c}
0 \\
\int_{\partial \Omega} \chi_{1} \partial_{2} \Psi_{j} d \sigma
\end{array}\right)\right\} \\
& +\sum_{2 \leqslant j \leqslant m} w_{j}\left\{l_{1}\left(\begin{array}{c}
0 \\
\int_{\partial \Omega} \chi_{j} \partial_{2} \Phi_{1} d \sigma
\end{array}\right)+l_{2}\left(\begin{array}{c}
\int_{\partial \Omega} \chi_{j} \partial_{1} \Phi_{2} d \sigma \\
0
\end{array}\right)+r\left(\begin{array}{c}
\int_{\partial \Omega} \chi_{j} \partial_{1} \Phi_{3} d \sigma \\
0
\end{array}\right)\right. \\
& \left.+w_{1}\left(\begin{array}{c}
0 \\
\int_{\partial \Omega} \chi_{j} \partial_{2} \Psi_{1} d \sigma
\end{array}\right)+\sum_{2 \leqslant k \leqslant m} w_{k}\left(\begin{array}{c}
\int_{\partial \Omega} \chi_{j} \partial_{1} \Psi_{k} d \sigma \\
0
\end{array}\right)\right\}
\end{aligned}
$$

We notice that the functions $\nabla \Phi_{1} \cdot y^{\perp}, \nabla \Psi_{1} \cdot y^{\perp}$ (resp. $\nabla \Phi_{2} \cdot y^{\perp}, \nabla \Phi_{3} \cdot y^{\perp}, \nabla \Psi_{2} \cdot y^{\perp}$ ) are odd (resp. even) with respect to the transformation $y \rightarrow y^{\prime}$. It follows that

$$
\begin{aligned}
\int_{\partial \Omega} & \left(\sum_{1 \leqslant j \leqslant m} w_{j} \chi_{j}\right) v \cdot y^{\perp} d \sigma \\
= & \sum_{1 \leqslant j \leqslant m} w_{j}\left(\sum_{1 \leqslant i \leqslant 2} l_{i} \int_{\partial \Omega} \chi_{j} \nabla \Phi_{i} \cdot y^{\perp} d \sigma+r \int_{\partial \Omega} \chi_{j} \nabla \Phi_{3} \cdot y^{\perp} d \sigma+\sum_{1 \leqslant k \leqslant m} w_{k} \int_{\partial \Omega} \chi_{j} \nabla \Psi_{k} \cdot y^{\perp} d \sigma\right) \\
= & w_{1}\left(l_{2} \int_{\partial \Omega} \chi_{1} \nabla \Phi_{2} \cdot y^{\perp} d \sigma+r \int_{\partial \Omega} \chi_{1} \nabla \Phi_{3} \cdot y^{\perp} d \sigma+\sum_{2 \leqslant k \leqslant m} w_{k} \int_{\partial \Omega} \chi_{1} \nabla \Psi_{k} \cdot y^{\perp} d \sigma\right) \\
& +\sum_{2 \leqslant j \leqslant m} w_{j}\left(l_{1} \int_{\partial \Omega} \chi_{j} \nabla \Phi_{1} \cdot y^{\perp} d \sigma+w_{1} \int_{\partial \Omega} \chi_{j} \nabla \Psi_{1} \cdot y^{\perp} d \sigma\right) .
\end{aligned}
$$


Gathering together (5.9)-(5.10) and (5.18)-(5.31), we derive the following system for the controlled dynamics of the rigid body.

$$
\begin{aligned}
& m \dot{l}_{1}=m r l_{2}-\left\{\dot{l}_{1} \int_{\partial \Omega} \Phi_{1} n_{1}+\dot{w}_{1} \int_{\partial \Omega} \Psi_{1} n_{1}\right. \\
& +r\left(l_{2} \int_{\partial \Omega} y_{1} \nabla \Phi_{2} \cdot \tau+r \int_{\partial \Omega} y_{1} \nabla \Phi_{3} \cdot \tau+\sum_{2 \leqslant k \leqslant m} w_{k} \int_{\partial \Omega} y_{1} \nabla \Psi_{k} \cdot \tau\right) \\
& +w_{1}\left(l_{1} \int_{\partial \Omega} \chi_{1} \partial_{1} \Phi_{1}+w_{1} \int_{\partial \Omega} \chi_{1} \partial_{1} \Psi_{1}\right) \\
& \left.+\sum_{2 \leqslant j \leqslant m} w_{j}\left(l_{2} \int_{\partial \Omega} \chi_{j} \partial_{1} \Phi_{2}+r \int_{\partial \Omega} \chi_{j} \partial_{1} \Phi_{3}+\sum_{2 \leqslant k \leqslant m} w_{k} \int_{\partial \Omega} \chi_{j} \partial_{1} \Psi_{k}\right)\right\}, \\
& m i_{2}=-m r l_{1}-\left\{i_{2} \int_{\partial \Omega} \Phi_{2} n_{2}+\dot{r} \int_{\partial \Omega} \Phi_{3} n_{2}+\sum_{2 \leqslant j \leqslant m} \dot{w}_{j} \int_{\partial \Omega} \Psi_{j} n_{2}\right. \\
& +r\left(l_{1} \int_{\partial \Omega} y_{2} \nabla \Phi_{1} \cdot \tau+w_{1} \int_{\partial \Omega} y_{2} \nabla \Psi_{1} \cdot \tau\right) \\
& +w_{1}\left(l_{2} \int_{\partial \Omega} \chi_{1} \partial_{2} \Phi_{2}+r \int_{\partial \Omega} \chi_{1} \partial_{2} \Phi_{3}+\sum_{2 \leqslant k \leqslant m} w_{k} \int_{\partial \Omega} \chi_{1} \partial_{2} \Psi_{k}\right) \\
& \left.+\sum_{2 \leqslant j \leqslant m} w_{j}\left(l_{1} \int_{\partial \Omega} \chi_{j} \partial_{2} \Phi_{1}+w_{1} \int_{\partial \Omega} \chi_{j} \partial_{2} \Psi_{1}\right)\right\}
\end{aligned}
$$

$$
\begin{aligned}
J \dot{r}= & \left\{l_{2} \int_{\partial \Omega} \Phi_{2} y^{\perp} \cdot n+\dot{r} \int_{\partial \Omega} \Phi_{3} y^{\perp} \cdot n+\sum_{2 \leqslant j \leqslant m} \dot{w}_{j} \int_{\partial \Omega} \Psi_{j} y^{\perp} \cdot n\right. \\
& -l_{1}\left(l_{2} \int_{\partial \Omega} y_{1} \nabla \Phi_{2} \cdot \tau+r \int_{\partial \Omega} y_{1} \nabla \Phi_{3} \cdot \tau+\sum_{2 \leqslant k \leqslant m} w_{k} \int_{\partial \Omega} y_{1} \nabla \Psi_{k} \cdot \tau\right) \\
& -l_{2}\left(l_{1} \int_{\partial \Omega} y_{2} \nabla \Phi_{1} \cdot \tau+w_{1} \int_{\partial \Omega} y_{2} \nabla \Psi_{1} \cdot \tau\right) \\
& +w_{1}\left(l_{2} \int_{\partial \Omega} \chi_{1} \Phi_{2} \cdot y^{\perp}+r \int_{\partial \Omega} \chi_{1} \nabla \Phi_{3} \cdot y^{\perp}+\sum_{2 \leqslant k \leqslant m} w_{k} \int_{\partial \Omega} \chi_{1} \nabla \Psi_{k} \cdot y^{\perp}\right) \\
& \left.+\sum_{2 \leqslant j \leqslant m} w_{j}\left(l_{1} \int_{\partial \Omega} \chi_{j} \nabla \Phi_{1} \cdot y^{\perp}+w_{1} \int_{\partial \Omega} \chi_{j} \nabla \Psi_{1} \cdot y^{\perp}\right)\right\} .
\end{aligned}
$$

This gives the result.

\subsection{Proof of Proposition 2.12.}

Let $\mathcal{R}=\operatorname{Span}\left\{\phi\left(T, t_{0}\right) \mathcal{M}_{i}\left(t_{0}\right) U ; U \in \mathbb{R}^{m}, i \geqslant 0\right\}$. To prove that $\mathcal{R}_{T}(\mathcal{A}, \mathcal{B})=\mathcal{R}$, we have to check that (i) $\mathcal{R}_{T}(\mathcal{A}, \mathcal{B})^{\perp} \subset \mathcal{R}^{\perp}$ and that (ii) $\mathcal{R}^{\perp} \subset \mathcal{R}_{T}(\mathcal{A}, \mathcal{B})$. 
(i) Pick any $p \in \mathcal{R}_{T}(\mathcal{A}, \mathcal{B})^{\perp}$. We aim to show that $p \in \mathcal{R}^{\perp}$, i.e. that $p^{*} \phi\left(T, t_{0}\right) \mathcal{M}_{i}\left(t_{0}\right)=$ 0 for all $i \geqslant 0$. For any $x \in \mathcal{R}_{T}(\mathcal{A}, \mathcal{B})$ written as $x=\int_{0}^{T} \phi(T, t) B(t) u(t) d t$ with $u \in$ $L^{2}\left(0, T, \mathbb{R}^{m}\right)$, we have that

$$
0=p^{*} x=\int_{0}^{T} p^{*} \phi(T, t) B(t) u(t) d t .
$$

This yields $p^{*} \phi(T, t) B(t) \equiv 0$. We claim the following: for all $i \geqslant 0$, we have

$$
p^{*} \phi(T, t) \mathcal{M}_{i}(t) \equiv 0 \text {. }
$$

We prove this claim by induction on $i$. For $i=0,(5.35)$ is obvious, since $\mathcal{M}_{0}(t)=B(t)$. Assume that (5.35) is true for $i-1$. Derivating with respect to $t$ in $p^{*} \phi(T, t) \mathcal{M}_{i-1}(t)=0$ yields

$$
0=p^{*} \phi(T, t)\left(-\mathcal{A}(t) \mathcal{M}_{i-1}(t)+\dot{\mathcal{M}}_{i-1}(t)\right)=p^{*} \phi(T, t) \mathcal{M}_{i}(t) .
$$

The claim is proved, and we infer that $p \in \mathcal{R}^{\perp}$.

(ii) Let $p \in \mathcal{R}^{\perp}$. From the proof of (5.35), we infer that

$$
\frac{d^{i}}{d t^{i}}\left[p^{*} \phi(T, t) B(t)\right]_{\mid t=t_{0}}=p^{*} \phi\left(T, t_{0}\right) \mathcal{M}_{i}\left(t_{0}\right)=0 \quad \forall i \geqslant 0 .
$$

Thus $t \mapsto p^{*} \phi(T, t) B(t)$ vanishes everywhere, by analyticity. It follows that

$$
\int_{0}^{T} p^{*} \phi(T, t) B(t) u(t) d t=0 \quad \text { for all } u \in L^{2}\left(0, T, \mathbb{R}^{m}\right),
$$

that is $p \in \mathcal{R}_{T}(A, B)^{\perp}$.

\section{ACKNOWLEDGEMENTS}

The authors wish to thank Institut Henri Poincaré (Paris, France) for providing a very stimulating environment during the "Control of Partial Differential Equations and Applications" program in the Fall 2010. The authors were partially supported by the Agence Nationale de la Recherche, Project CISIFS, grant ANR-09-BLAN-0213-02.

\section{REFERENCES}

[1] C. Amrouche, V. Girault, and J. Giroire. Dirichlet and Neumann exterior problems for the ndimensional Laplace operator: an approach in weighted Sobolev spaces. J. Math. Pures Appl. (9), 76(1):55-81, 1997.

[2] Thomas Chambrion and Mario Sigalotti. Tracking control for an ellipsoidal submarine driven by Kirchhoff's laws. IEEE Trans. Automat. Control, 53(1):339-349, 2008.

[3] Jean-Yves Chemin. Fluides parfaits incompressibles. Astérisque, (230):177, 1995.

[4] C. Conca, M. Malik, and A. Munnier. Detection of a moving rigid body in a perfect fluid. Inverse Problems, 26:095010, 2010.

[5] Carlos Conca, Patricio Cumsille, Jaime Ortega, and Lionel Rosier. On the detection of a moving obstacle in an ideal fluid by a boundary measurement. Inverse Problems, 24(4):045001, 18, 2008.

[6] Jean-Michel Coron. On the controllability of 2-D incompressible perfect fluids. J. Math. Pures Appl. (9), 75(2):155-188, 1996.

[7] Jean-Michel Coron. Control and nonlinearity, volume 136 of Mathematical Surveys and Monographs. American Mathematical Society, Providence, RI, 2007.

[8] Michel Fliess, Jean Lévine, Philippe Martin, and Pierre Rouchon. Flatness and defect of nonlinear systems: introductory theory and examples. 31:1327-1361, 1995.

[9] Olivier Glass. Exact boundary controllability of 3-D Euler equation. ESAIM Control Optim. Calc. Var., 5:1-44 (electronic), 2000.

[10] Olivier Glass, Franck Sueur, and Takéo Takahashi. Smoothness of the motion of a rigid body immersed in an incompressible perfect fluid. preprint, arXiv:1003.4172. 
[11] V. I. Judovič. A two-dimensional non-stationary problem on the flow of an ideal incompressible fluid through a given region. Mat. Sb. (N.S.), 64 (106):562-588, 1964.

[12] A. V. Kazhikhov. Note on the formulation of the problem of flow through a bounded region using equations of perfect fluid.

[13] Keisuke Kikuchi. Exterior problem for the two-dimensional Euler equation. J. Fac. Sci. Univ. Tokyo Sect. IA Math., 30(1):63-92, 1983.

[14] Horace Lamb. Hydrodynamics. Cambridge Mathematical Library. Cambridge University Press, Cambridge, sixth edition, 1993. With a foreword by R. A. Caflisch [Russel E. Caflisch].

[15] Naomi Ehrich Leonard. Stability of a bottom-heavy underwater vehicle. Automatica J. IFAC, 33(3):331-346, 1997.

[16] Naomi Ehrich Leonard and Jerrold E. Marsden. Stability and drift of underwater vehicle dynamics: mechanical systems with rigid motion symmetry. Phys. D, 105(1-3):130-162, 1997.

[17] N. G. Lloyd. Degree theory. Cambridge University Press, Cambridge, 1978. Cambridge Tracts in Mathematics, No. 73.

[18] Jaime H. Ortega, Lionel Rosier, and Takéo Takahashi. Classical solutions for the equations modelling the motion of a ball in a bidimensional incompressible perfect fluid. M2AN Math. Model. Numer. Anal., 39(1):79-108, 2005.

[19] Jaime H. Ortega, Lionel Rosier, and Takéo Takahashi. On the motion of a rigid body immersed in a bidimensional incompressible perfect fluid. Ann. Inst. H. Poincaré Anal. Non Linéaire, 24(1):139-165, 2007.

[20] Carole Rosier and Lionel Rosier. Smooth solutions for the motion of a ball in an incompressible perfect fluid. J. Funct. Anal., 256(5):1618-1641, 2009.

[21] J. Simon. Compact sets in the space $L^{p}(0, T ; B)$. Ann. Mat. Pura Appl. (4), 146:65-96, 1987.

[22] Eduardo D. Sontag. Mathematical control theory, volume 6 of Texts in Applied Mathematics. SpringerVerlag, New York, 1990. Deterministic finite-dimensional systems.

[23] Elias M. Stein. Singular integrals and differentiability properties of functions. Princeton Mathematical Series, No. 30. Princeton University Press, Princeton, N.J., 1970.

Current address: CEREMADE, Université Paris-Dauphine, Place du Maréchal de Lattre de Tassigny, 75775 Paris Cedex 16, France

E-mail address: glass@ceremade.dauphine.fr

Current address: Institut Elie Cartan, UMR 7502 UHP/CNRS/INRIA, B.P. 70239, F-54506 Vandœuvrelès-Nancy Cedex, France

E-mail address: rosier@iecn.u-nancy.fr 